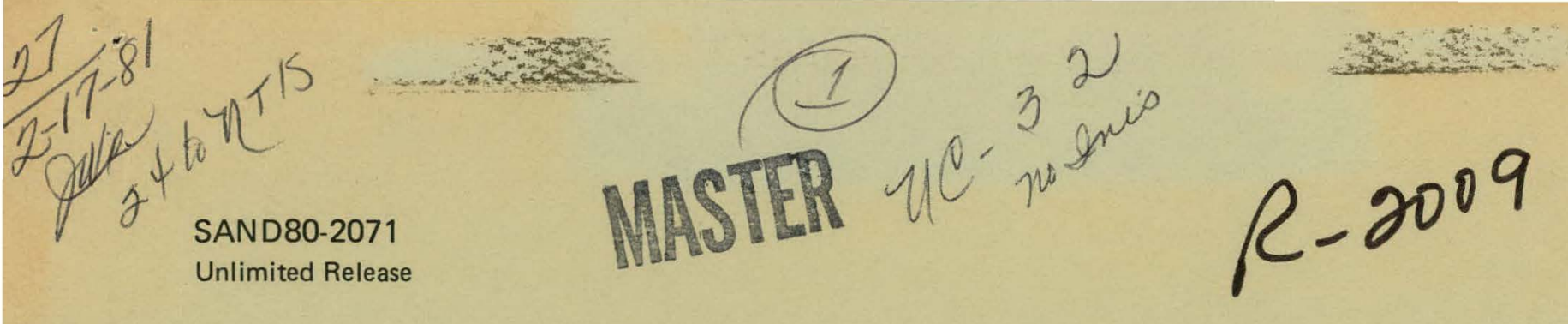

\title{
Deconvolution Using the Complex Cepstrum
}

\author{
H. Bryan Riley
}

Prepared by Sandia National Laboratories, Albuquerque. New Mexico 87185 and Livermore, California 94550 for the United States Department of Energ under Contract DE-AC04-76DP00789

Printed December 1980

\section{in Sandia National Laboratories}




\section{DISCLAIMER}

This report was prepared as an account of work sponsored by an agency of the United States Government. Neither the United States Government nor any agency Thereof, nor any of their employees, makes any warranty, express or implied, or assumes any legal liability or responsibility for the accuracy, completeness, or usefulness of any information, apparatus, product, or process disclosed, or represents that its use would not infringe privately owned rights. Reference herein to any specific commercial product, process, or service by trade name, trademark, manufacturer, or otherwise does not necessarily constitute or imply its endorsement, recommendation, or favoring by the United States Government or any agency thereof. The views and opinions of authors expressed herein do not necessarily state or reflect those of the United States Government or any agency thereof. 


\section{DISCLAIMER}

Portions of this document may be illegible in electronic image products. Images are produced from the best available original document. 
Issued by Sandia National Laboratories, operated for the United States Department of Energy by Sandia Corporation.

NOTICE : This report was prepared as an account of work sponsored by an agency of the United States Government. Neither the United States Government nor any agency thereof, nor any of their employees, nor any of their contractors, subcontractors, or their employees, makes any warranty, express or implied, or assumes any legal liability or responsibility for the accuracy, completeness, or usefulness of any information, apparatus, product, or process disclosed, or represents that its use would not infringe privately owned rights. Reference herein to any specific commercial product, process, or service by trade name, trademark, manufacturer, or otherwise, does not necessarily constitute or imply its endorsement, recommendation, or favoring by the United States Government, any agency thereof or any of their contractors or subcontractors. The views and opinions expressed herein do not necessarily state or reflect those of the United State Government, any agency thereof or any of their contractors or subcontractors.

Printed in the United States of America Available from

National Technical Information Service

U. S. Department of Commerce

5285 Port Royal Road

Springfield, VA 22161

NTIS price codes

Printed copy: $\$ 6.00$

Microfiche copy: A01 


\section{PAGES 1 to 2 WERE INTENTIONALLY LEFT BLANK}


SAND80-2071

Unlimited Releașe

Printed December 1980

Distribution

Category UC-32

DECONVOLUTION USING THE COMPLEX CEPSTRUM

H. Bryan Riley

Ground Motion and Seismic Division 1111

Sandia National Laboratories

Albuquerque, NM 87185

\section{ABSTRACT}

This paper presents the theory, description, and impleion of a generalized linear filtering system for the nontation filtering of convolved signals. It is a detailed lo problems and requirements associated with look at the prution of signal components. Related properthe deconvolution of synthetic example is shown ties are also developed. A synthetic
and is followed by ap aplication using real seismic data.

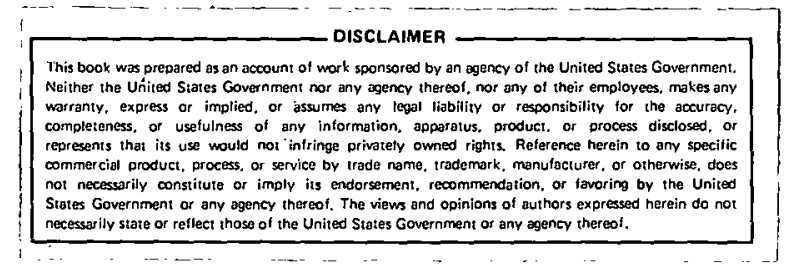

"Summer employee, 1980 
The author expresses sincere gratitude for the patience and guidance of Professor W. E. Alexander at North Carolina Agricultural and Technical State University and S. D. Stearns at Sandia National Laboratories. The author thanks Luke Vortman of Sandia National Laboraturies for supplying and analyzing the seismic data; and Dr. Ward Collis and E. E. Sherrod at NC A\&T State University and T. S. McDonald and J. W. Garner of the Dikewood Corporation for their useful suggestions and criticisms. Thanks also goes to the management and oupcrvisury per'sonnel at Sandia National Laboratories for the opportunity to use their farilitios: 


\section{CONTENTS}

List of Symbols

1. Introduction $\quad$. $\quad$. $\quad 9$

1. 1 Advantages of Homomorphic Deconvolution . . . . . 9

1. 2 Problem Definition . . . . $\quad 10$

2. Homomorphic Systems $\quad$ $\quad$. . . . . 11

2.1 Introduction $\quad . \quad \ldots \ldots . .11$

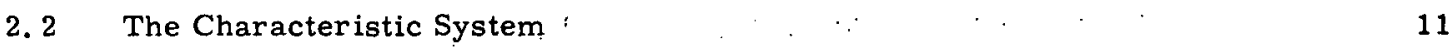

2. 3 The Inverse Characteristic System $\quad$ * $\quad$ : $\quad$. 13

2.4 The Linear System ․ . . . . . . $\quad 14$

3. Some Properties of the Complex Cepstrum .

3.1 Real Cepstrum : . $\quad$ : $\quad 15$

3. 2 A Convolution Becomes a Sum ? $\quad 16$

$\begin{array}{llll}3.3 & \text { Cepstrum of an Impulse } & 17\end{array}$

3.4 Scaling $\quad \cdot \cdot \cdot \cdot \cdot$

3. 5 Shifting $r$

3. 6 Other Aspects $\quad 21$

3.6.1 Causality 21

3.6.2 Input-Output Cepstral Relationship 23

4. Computing the Complex Cepstrum 24

4.1 Introduction $\quad . \quad 24$

4 Input Normalization and Requirements 2 : . . . 25

4. 3 Hhase Unwrapping . $\quad$. . . . . 25

$\begin{array}{lll}\text { 4. } 4 \text { The Liriear Phase Component } & 28\end{array}$

5. Deconvolution Using a Known Waveform $\quad$ - $\quad 29$

5.1 Introduction $1 \cdot \cdot \cdot 29$

5. 2 Fabrication of the Input $\quad \cdots$

5. 3 The Computed Complex Cepstrum 30

5. 4 Linear Filtering $\quad \cdot \quad 31$

5: 5 Results $\quad 32$

6. A Computation With Real Data 33

6. 1 Introduction and Background 33

6. 2 Procedure of Computation . 33

$\begin{array}{lll}6.3 & \text { Results and Conclusions } & 37\end{array}$

References $\quad 39$ 


\section{ILLUSTRATIONS}

\begin{tabular}{|c|c|c|}
\hline Figure & & Page \\
\hline 1 & The Generalized Filtering System & 10 \\
\hline 2 & A Simple Model for Seismic Waveform & 11 \\
\hline 3 & The Cascade Representation of the Characteristic System $D_{*}$ & 12 \\
\hline 4 & The Cascade Representation of the Inverse Characteristic System $D_{*}^{-1}$ & 14 \\
\hline 5 & A Representation of the Linear System & 14 \\
\hline c & A Șequence of Two Impulses & 17 \\
\hline 7 & The Computed Complex Cepstrum & 18 \\
\hline 8 & Detailed Homomorphic System for Deconvolution & 24 \\
\hline 9 & Homomorphic System for Oporation uil a Maximüm- or Mired-Phaso Scquence & 36 \\
\hline 10 & Input Waveform to the Homomorphic System & 27 \\
\hline 11 & Principal Value of the Phase & 27 \\
\hline 12 & The Unwrapped Phase With the Linear-Phase Cumpunent Removed & 28 \\
\hline 13 & Decaying Sine Wave & 29 \\
\hline 14 & Sequence of Two Impulses. & 29 \\
\hline 15 & Convolutional Result & 29 \\
\hline 16 & Coimputed Complex Cepstrum & 30 \\
\hline 17 & Low-Time Cepstral Component & 31 \\
\hline 18 & High-Time Cepstral Component & 31 \\
\hline 19 & Reconstructed Waveform for the Low-Time Component & 32 \\
\hline 20 & Reconstructed Waveform for the High-Time Component & 32 \\
\hline 21 & Unfiltered Seismic Signal & 33 \\
\hline 22 & The Power Spectrum at the Seismic Input & 34 \\
\hline 23 & Low-Pass Butterworth Type Filter & 35 \\
\hline 24 & Low-Pass Filtered Input Waveform & 35 \\
\hline 25 & Computed Complex Cepstrum & 36 \\
\hline 26 & Low-Time Cepstral Component & 36 \\
\hline 27 & High-Time Cepstral Component & 37 \\
\hline 28 & Computed Source Wavelet & 38 \\
\hline 29 & Computeril Inpuloo Rcsponse of the Medium 'Traveled & 38 \\
\hline
\end{tabular}


List of Symbols

$\begin{array}{ll}+ & \text { Addition } \\ * & \text { Multiplication } \\ \mathbf{x}(\mathrm{n}) & \text { Convolution } \\ \mathrm{nx}(\mathrm{n}) & \text { A Real Function of Time } \\ \hat{\mathbf{x}}(\mathrm{n}) & \text { Cerivative of the Real Function of Time } \\ \mathrm{D}_{*} & \text { Characteristic System } \\ \mathrm{D}_{*}^{-1} & \text { Inverse Characteristic System } \\ \mathrm{L} & \text { Linear System } \\ \overline{\mathrm{L}} & \text { Complex Logarithm } \\ \alpha & \text { Weighting Factor } \\ \overline{\mathrm{X}} & \text { Discrete Fourier Transform }\end{array}$




\section{DI:CONVOLUTTION USING THE COMPLEX CEPSTRUM}

1. Introduction

Many signals are composed of the convolution of two or more signals. A nonlinear filtering system called the homomorphic system ${ }^{1}$ is useful in separating signals that have been combined through convolution. An investigation of deconvolution using this technique will be the focal topic of this paper.

This investigation will be limited to the application of homomorphic deconvolution to seismic signals. A seismic record is often represented as the convolution of a seismic wavelet with the impulse response of the transmission path. Deconvolution shall be defined here as an operation that unfolds the recorded seismic data. The final results yield the seismic-source wavelet separated from the impulse response of the medium traveled.

The recovered seismic wavelet is of importance in studies of elastic wave attenuation and dispersion in the earth. Also, the impulse response of the medium traveled offers useful information in geological studies. The separated components are needed to make conclusions about the seismic event.

\section{1 Advantages of Homomorphic Deconvolution.}

Homomorphic deconvolution offers substantial advantages over several other systems in separating signal components. Research of the homomorphic system was undertaken because deconvolution may be accomplished with little prior knowledge of the signal components. Little or no information is usually available about the signal components of a seismic event; however, if one of the components is previously defined, then the other one may be recovered by simple filtering.

The design of an inverse filtering system for deconvolution requires a detailed knowledge of at least one signal component. This requirement generally eliminates the use of an inverse filtering system to separate the signal components of a seismic event.

Deconvolution may also be performed with the use of the optimum zero-lag Weiner filtering. This process is not practical for seismic data because the impulse response of the medium or the shape of the seismic wavelet to be removed must be known. Another requirement is that the wavelet be minimum phase. 


\subsection{Problem Definition}

It is known that the recorded seismic trace is not the result of linearly combining the signal components; thus a linear filter cannot he used for scparating the components. A nonlinear filtering system must be used for separating such components. The homomorphic system for deconvolution is based on a generalization of the linearity property. It is a nonlinear filtering system expressed in a linear fashion. 2 The primary reason for using this approach is that linear systems are relatively easy to implement. They also give excellent results when separating signal components that have been combined in a linear fashion. The investigated system transforms the signal from a convolutional space to an additive space so that linear filtering techniques can be applied.

The oygteni alsu ulueys a generalized principle of the superposition theorem. The operation of the nonlinear system as described horein is designed to malch that of a linear system. A nonlinear operation to separate convolved components corresponds to a linear opcration to separate added components, This general operation is depictod in Figure 1.

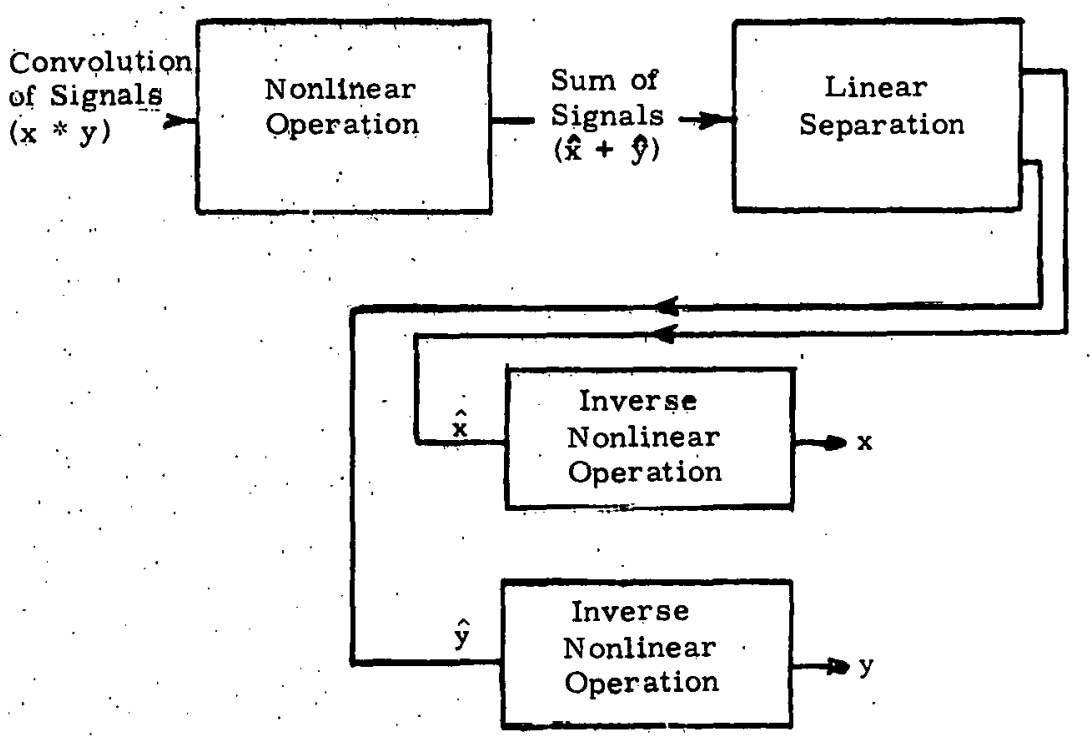

Figure 1. The Generalized Filtering Syatem 


\section{Homomorphic Systems}

\subsection{Introduction}

Seismic records are recorded in a reverberant environment. 'The signal is considered as the sum of a number of overlapping delayed replicas of some basic source waveform. The overlapping waveforms are disguised with an impulse train component which contains information about the earth's medium. The reverberation is taken as distortion and the objective is to recover separately the basic source wavelet from the impulse train. A simple model is shown in Figure 2 with $s(n)$ denoting the source wavelet and $t(n)$ denoting the transmission path.

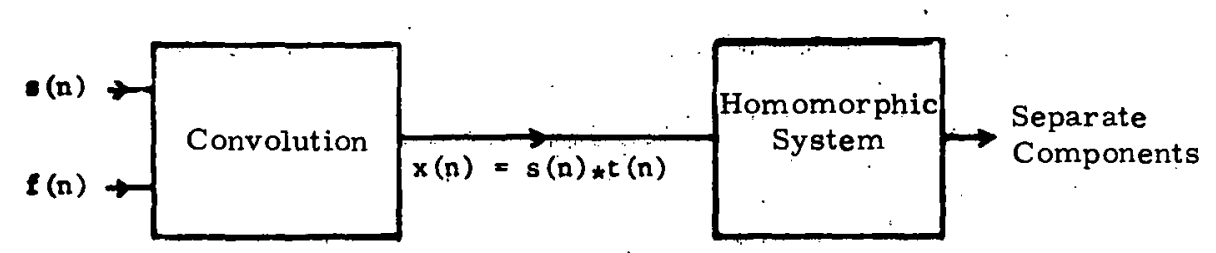

Figure 2. A Simple Model for Seismic Waveform

The overall system is implemented in three major subsystems. The theory of each subsystem is developed in the following sections.

\subsection{The Characteristic System}

The notation $D_{*}$ will be used to refer to the characteristic system. The Z-transform and Discrete Fourier Transform (DFT) will be used interchangeably at times for convenience of discussion. The DFT is also used because computations are performed on sampled data. This is valid because each may be obtained from the other by a simple change in variables. The rclationship is $Z=e^{j \omega T}$ where $T$ is the sampling interval, $Z$ is the $Z$-transform variable, and $\omega$ is the DFT variable. The DFT is referred to specifically in reference to computations and is implemented with a Faot Fourier Transform (FFT) algorithm,

The function of $D_{*}$ is to transform the signal from a convolution space to an additive space. This is given by the relationship

$$
\begin{aligned}
D_{*}[X(n)] & =D_{*}\left[x_{1}(n) * x_{2}(n)\right] \\
& =D_{*}\left[x_{1}(n)\right]+D_{*}\left[x_{2}(n)\right] \\
& =\hat{x}_{1}(n)+\hat{x}_{2}(n)=\hat{x}(n)
\end{aligned}
$$

The key to this mathematical representation is that the Z-transform of the convolution of two sequences is equal to the product of the $\mathrm{Z}$-transforms. The logarithmic function is employed becáuse it separates a product. into a sum. 
In terms of the $Z$-transform, the following equations give the transformation from a convolutional input to an additive output.

$$
X(z)=\sum_{n=0}^{N-1} x(n) z^{-n} \text {, }
$$

where

$$
\begin{aligned}
z= & e^{j \omega T} \\
\tilde{X}(z) & =\log [x(z)] \\
& =\log |X(z)|+j \arg [X(z)] \\
\hat{x}(n) & =\frac{1}{2 \pi j} \int \tilde{X}(z) z^{n \sim 1} d z:
\end{aligned}
$$

These equations define the quantity complex cepstrum for uniformity samplcd discrele functions. The complex cepstrum is a real-time series. The $Z$-transform is mentioned because it is an important tool used in the analysis and representation of the discrete time-invariant systems. The DFT is used to calculate the $Z$-transform by a change in variables $\left(Z=e^{j \omega T}\right)$.

The characteristic system computes the complex cepstrum, $x(n)$, and is accomplished by three subsystems in cascade, It is depicted in Figure 3.

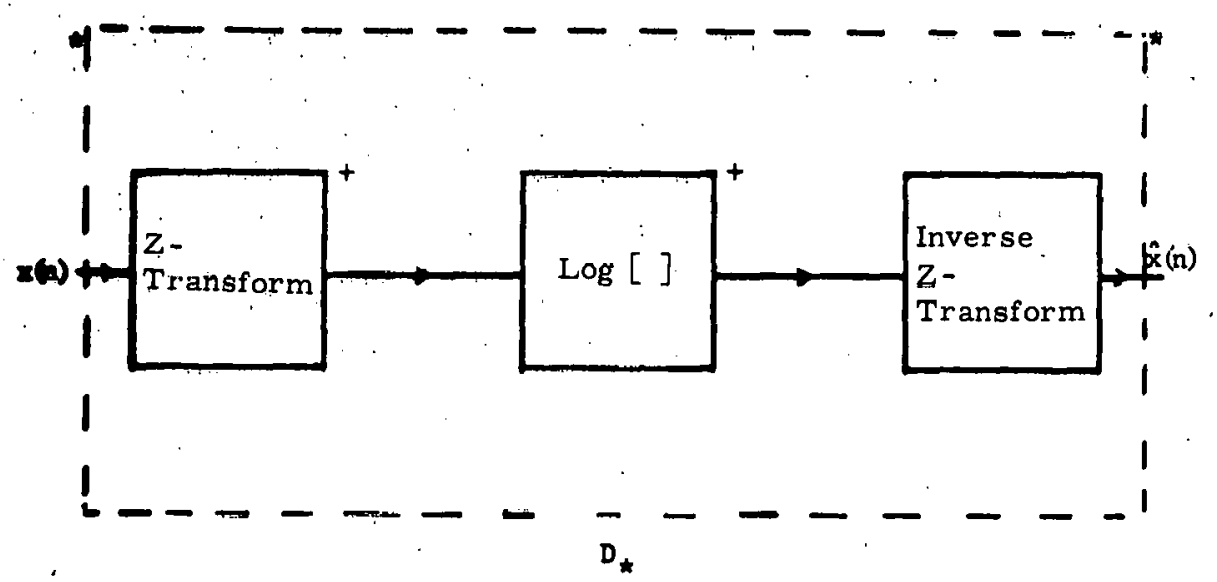

Figure 3. The Cascade Representation of the Characteristic System $D_{*}$

The word "cepstrum" originates from the work of Bogert et al (1963). ${ }^{3}$ (The term is an anagram of the word spectrum.) Cepstrum is defined as the power spectrum of the logarithm of the signal power spectrum. "Complex" was added by Oppenheim et al to emphasize the point that the cepstrum is computed retaining both amplitude and phase information. ${ }^{1}$ The next step is to pass the cepstral quality through the linear filtering system. 
There is an important consideration in computing the complex cepstrum quantity. This output sequence from the chi,racteristic system must be nonambiguous and unique. This requirement must . be met so that the seq:ence can be inverse transformed to give a real-valued complex cepstrum. A more detailed discussion of this and other considerations is pnesented in Section 3.

\section{3 The Inverse Characteristic System}

The inverse characteristic system is also fixed and will be denoted by $D_{*}^{-1}$. This system is composed of three subsystems in cascade and is designed to reverse the operations of the characteristic system. This stage precedes the linear filtering system and simply transforms the additive space back into a convolutional space.

The inverse characteristic system is alpo based upon the generalized principle of the superposition theorem. It is defined by the relationship

$$
\begin{aligned}
D_{*}^{-1}[\hat{x}(n)] & =D_{*}^{-1}\left[\hat{x}_{1}(n)+\hat{x}_{2}(n)\right] \\
& =D_{*}^{-1}\left[\hat{x}_{1}(n)\right] * D_{*}^{-1}[\hat{x}(n)] \\
& =x_{1}(n) * x_{2}(n)=x(n)
\end{aligned}
$$

Defined in terms of the Z-transform, Eqs (6), (7), and (8) give the transformation from an additive input to a convolutional output.

$$
\begin{aligned}
& x(z)=\sum_{n=0}^{N-1} x(n) z^{-n} \\
& x(z)=\exp [x(z)] \\
& x(n)=\frac{1}{2 \pi j} \oint \tilde{X}(z) z^{n-1} d z .
\end{aligned}
$$

Each of the previous three equations' is represented in one of the blocks shown in Figure 4.

The inverse characteristic system is implemented computationally by means of the DFT. There is no computational uniqueness problem encountered in taking the complex exponential of the transformed sequence.

The output of this system will be one of the original signals convolutional components, a real-time sequence. The undesired components will have been filtered out by the preceding linear system. 


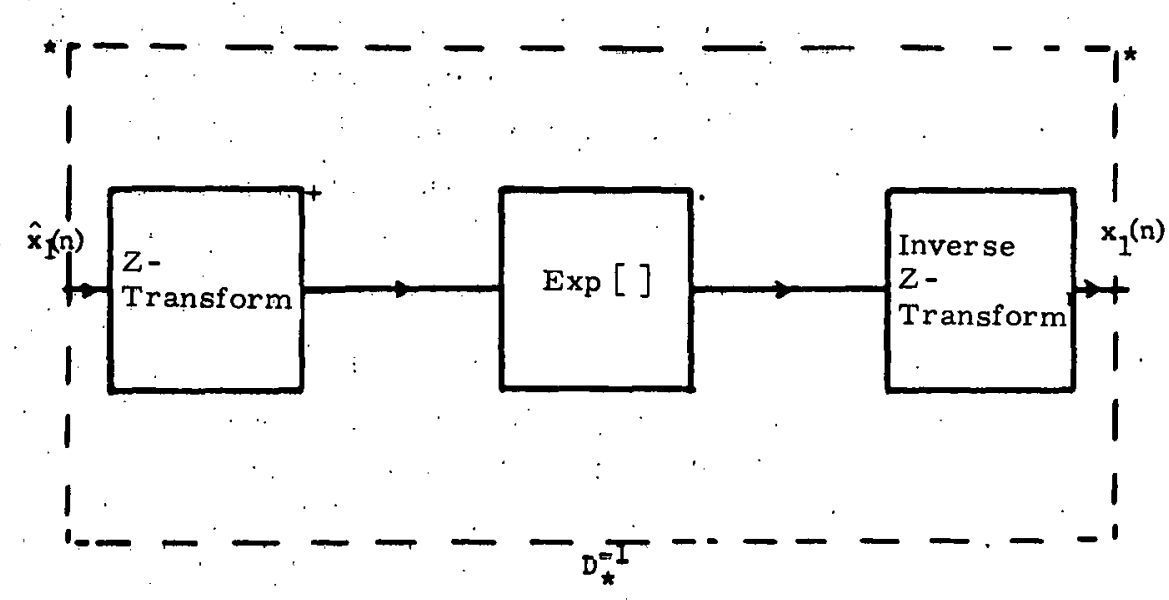

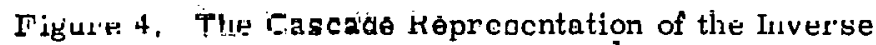
Characteristic System $\mathrm{D}_{*}^{-1}$

\subsection{The Linear System}

The linear filtering system follows the characteristic system and is denoted by L. The input of this subsystem is the complex cepstrum. which contains the additive contributions of the composite signal makeup.

Linear-filtering techniques are employed beçause linear filter behavior is known for separate components. Thus, the behavior of the generalized linear filter can be deduced as a result of the superposition theorem for separation of added components.

The conventional linear system can be expressed as

$$
\begin{aligned}
L[X(n)] & =L\left[x_{1}(n)+x_{2}(n)\right] \\
& =L\left[x_{1}(n)\right]+L\left[x_{2}(n)\right] \\
& =x_{1}^{\prime}(n)+x_{2}^{\prime}(n)=x^{\prime}(n) .
\end{aligned}
$$

If an input signal is composed of a linear combination of signals when the supperpesition principle is applied to linear systems, the output is a linear rnmhination of oorreoponding mit.mil. This is depicted in Figure 5.

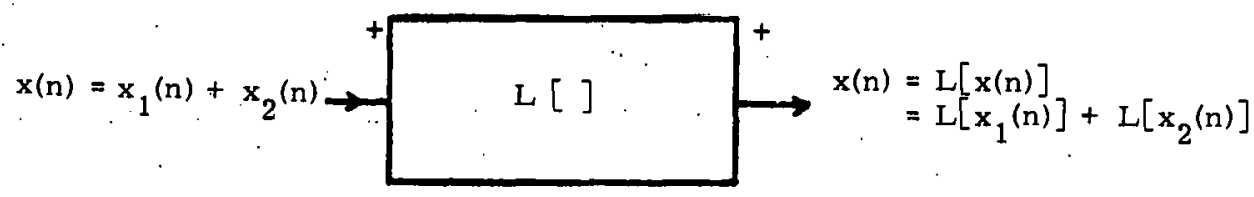

Figure 5. A Representation of the Linear System 
The overall homomorphic system is a filtering system with the property that one component passes through essentially unaltered, while the undesirable component is removed.

The structure of the complex cepstrum $\hat{\mathbf{x}}(n)$ is such that the impulse functions of the reflector series occupy separate time intervals. This phenomenon holds true for the deconvolution of speech or medical waveforms. Specifically for the seismic cepstrum, the source component will be concentrated abqut the origin; the impulse component of the medium will also be concentrated about the origin. The impulse component of the medium traveled will contribute to the cepstrum for times equal to and greater than that of the first reflection. 45

The tendency for the components to occupy different time intervals in the cepstral domain suggests the use of low-time and high-time frequency invariant filters. The frequency inyariant operators are referred to as cepstral gates or cepstral windows.

The source component has a relatively smooth log spectrum. Gating just the time near the origin of the cepstrum corresponds to șmoothing that spectrum. ${ }^{6}$ This results in retaining the source component. This output is now the input of the inverse characteristic system.

The deșign of the cepstral gate involves estimating the period of the reflector series or the first interarrival time of the impulse. The estimation is accomplished by either physical considerations or by observing this complex cepstral waveform. A high-time gate is placed around the remainder of the cepstrum where the same procedures and arguments are followed.

\section{Some Properties of the Complex Cepstrum}

This section is devoted to some inherent aspects of the complex cepstrum. All of the properties discussed are straightforward and are based on the established fundamental properties of the DFT and discrete systems. Several other properties not disçussed here can be commonly found in literature, and texts that discuss the complex cepstrum. $14 \%$

\section{1 Real Cepstrum}

The computed complex cepstrum of a real-time sampled sequence will also be a real-time sequence. The definition of the complex cepstrum is a good starting point to illustrate this property.

Consider the definition of the complex cepstrum written as the real part only:

$$
x_{m}=\sum_{n=0}^{N-1} \log \left|x_{n}\right| \cos \frac{2 \pi m n}{N}-\dot{\theta}_{n} \sin \frac{2 \pi m n}{N}
$$

where the log of the DFT is written in polar form, 


$$
\log \left[\left|\bar{x}_{n}\right| e^{j \theta n}\right]=\bar{L}_{n}
$$

Recall the property of a real spectrum,

$$
\overline{\mathrm{x}}_{\mathrm{N}-\mathrm{m}}=\overline{\mathrm{x}}_{\mathrm{m}}^{*}
$$

but

$$
\overline{\mathrm{L}}_{\mathrm{n}}=\log \left|\dot{\mathrm{x}}_{\mathrm{n}}\right|+j \theta \mathrm{n}
$$

where the real part has been ensured to be even and a periodic function of $\omega$; the imaginary part is odd and a periodic function of $\omega$. This satisfies the condition

$$
\overline{\mathrm{L}}_{\mathrm{N}-\mathrm{n}}=\overline{\mathrm{L}}_{1 \mathrm{n}}^{-*}
$$

and the $\operatorname{DFT}^{-1}\left[\overline{\mathrm{L}}_{n}\right]$ must be a real-time sequence. It can be concluded that the computed complex cepstrum is real for a real input: .

if

$$
\overline{\mathrm{x}}_{\mathrm{N}-\mathrm{m}}=\overline{\mathrm{x}}_{\mathrm{m}}
$$

then

$$
\hat{\mathrm{x}}_{\mathrm{N}-\mathrm{m}}=\hat{\mathrm{x}}_{\mathrm{m}}
$$

\section{2 A Conviolution Becomes a Sum}

The definition of the complex cepstrum allows a convolution input to be expressed as a cepstral sum output. The logarithmic operation is the hasis for this property. Lct $x_{m}$ and $f_{m}$ be two convolved components of an input $\mathrm{g}_{\mathrm{m}}$; then,

$$
\begin{aligned}
& g_{m}=\sum_{n=0}^{N-1} x_{m m-n}^{f} \\
& \bar{G}_{m}=\bar{x}_{m} \cdot \bar{F}_{m} \\
& \log \bar{G}_{m}=\log \bar{x}_{m}+\log \bar{F}_{m} .
\end{aligned}
$$

The cepstral output is now expressed in additive form:

$$
\hat{\mathrm{g}}_{\mathrm{m}}=\sum_{n=0}^{N-1}\left(\log X_{m}+\log F_{m}\right) e^{j 2 \pi m n / N}
$$




$$
\begin{aligned}
& \hat{g}_{m}=\sum_{n=0}^{N-1} \log x_{m} e^{j 2 \pi m n / N}+\sum_{n=0}^{N-1}\left(\log F_{m}\right) e^{j 2 \pi m n / N} \\
& \hat{g}_{m}=\hat{x}_{m} \because \hat{s}_{m} .
\end{aligned}
$$

\section{3 Cepstrum of An Impulse}

Consider a digital impulse defined as unit amplitude occurring at time zero.

$$
\begin{aligned}
& x_{m}=\begin{array}{l}
1 m=0 \\
0 \text { otherwise }
\end{array} \\
& \bar{x}_{m}=\sum_{M}^{N-1} x_{m} e^{-j 2 \pi m n / N} \quad m=0,1,2, \ldots, N-1 \\
& \log \bar{x}_{m}=0 \quad m=0,1,2, \ldots . N-1 \ldots
\end{aligned}
$$

Thus the cepstrum of a single impulse is zero,

For a sequence of impulses, results show that the computed complex cepstrum is another impulse train. It is found that there exists a relationship between the time occurrences of the impulse in the original input and the time occurrences of the impulse in the computed cepstral output.

To illustrate this properly, two impulses are spaced $1 \mathrm{~s}$ apart as shown in Figure 6. This is equivalent to the mathematical discrete set

$$
\mathrm{x}_{\mathrm{m}}=[1,0,0,0, \ldots 0,0.75, \ldots, \mathrm{N}]
$$

where $T=0.0267 \mathrm{~s}$ and the only nonzero samples are at $\mathrm{t} \doteq 0$ and $\mathrm{t} \doteq 1.0$ with sample values of 1.0 and 0.75 , respectively.

Figure 6

A Sequence of Two Impulses

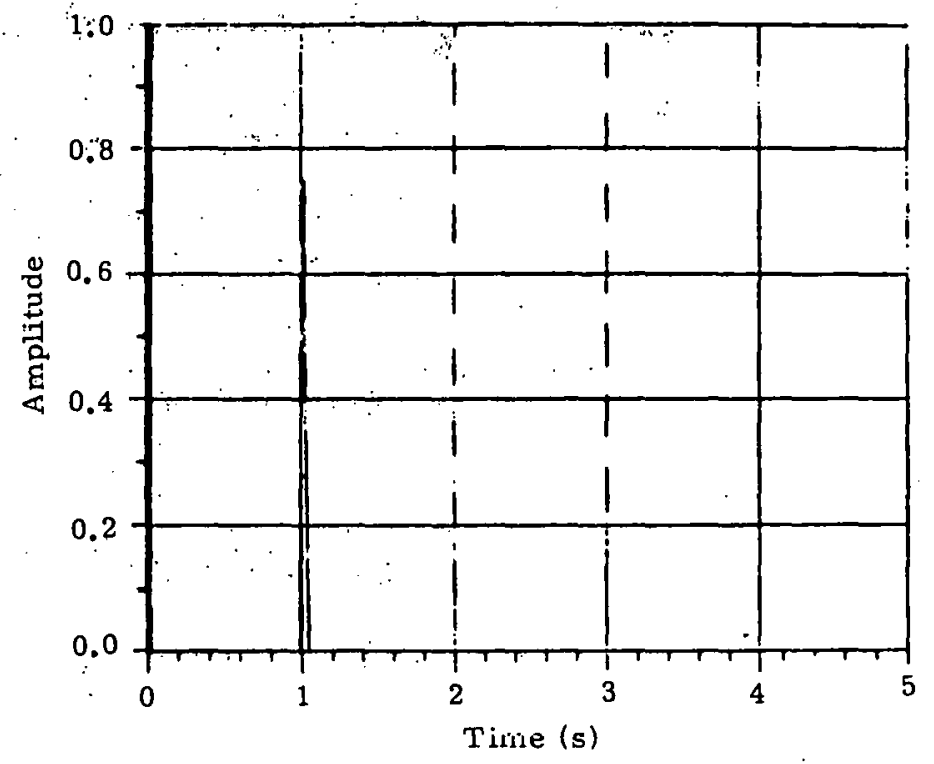


The DFT is

$$
\begin{aligned}
\bar{x}_{m} & =\sum_{n=0}^{N-1} x_{m} e^{-j 2 \pi n T / N} \\
& =a+b \exp ^{-j 2 \pi n T / N} ;|n| \leq \frac{n}{2} .
\end{aligned}
$$

The logarithm of $\overline{\mathrm{x}}_{\mathrm{m}}$ is given by

$$
\begin{gathered}
\log \left[\mathrm{x}_{\mathrm{m}}\right]=1 / 2 \log \left[\mathrm{a}^{2}+\mathrm{b}^{2}+2 a b \cos (2 \pi \mathrm{nT} / \mathrm{N})\right] \\
+\mathrm{j} \tan ^{-1} \frac{\mathrm{b} \sin \frac{2 \pi n T}{\mathrm{~N}}}{\mathrm{a} \operatorname{cus} \frac{2 \pi n T}{N}} \\
\ln \mid \leq \frac{N}{2}
\end{gathered}
$$

It can now be observed that, in the frequency, domain, both the real and imaginary parts repeat at $\mathrm{n}=\mathrm{N} / \mathrm{T}, 2 \mathrm{~N} / \mathrm{T}, 3 \mathrm{~N} / \mathrm{T}, \ldots$. Thus, since the cepstrum $\hat{\mathbf{x}}_{\mathrm{m}}$ is the inverse DFT of $\log \overline{\mathrm{X}}_{\mathrm{m}^{\circ}}$ it consists essentially of a șequence of impulses occurring at multiples $(\mathrm{m}=0, \mathrm{~T}, 2 \mathrm{~T}, 3 \mathrm{~T}, \ldots)$ of the original time spacing. Figure 7 shows the computed complex cepstrum.

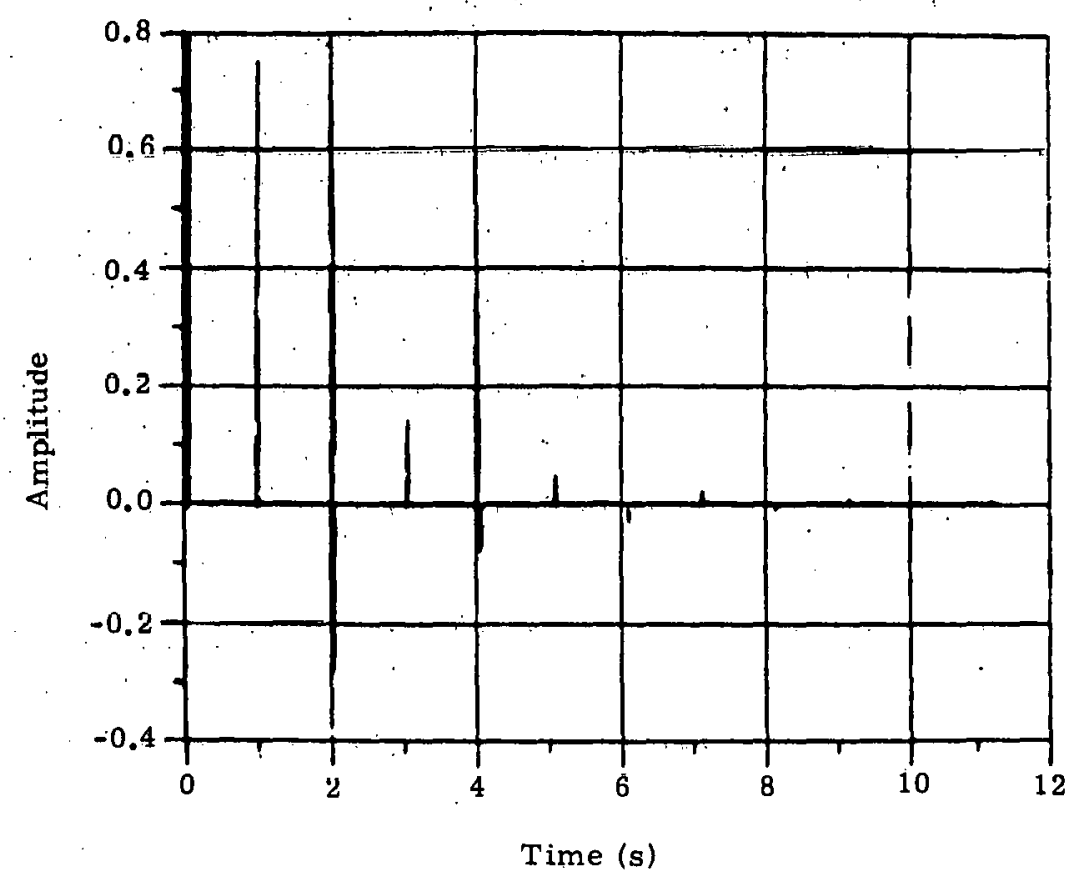

Figure 7. The Computed Complex Cepstrum 
This relationship holds for a more complicated input sequence of impulses. The time relationship between the two becomes more complex and difficult to envision.

\subsection{Scaling}

The input waveform is scaled for the purpose of investigating its effect on the complex cepstrum. A short mathematical development shows that only the first computed cepstral point is modified. The remainder of the points are essentially identical.

$$
\begin{aligned}
& \text { Let } \\
& \mathrm{x}_{\mathrm{m}}^{\prime}=A \mathrm{x}_{\mathrm{m}} ; \mathrm{A}>0 \\
& \operatorname{DFT}\left[\mathrm{x}_{\mathrm{m}}^{\prime}\right]=A \overline{\mathrm{X}}_{\mathrm{m}}^{\prime} \\
& \log \left[\bar{x}_{m}^{\prime}\right]=\log A+\log x_{m} \\
& x_{m}^{\prime}=\operatorname{IDFT}\left[\log \left[x_{m}^{\prime}\right]\right]=\log A \sum_{n=0}^{N-1} e^{j 2 m n / N} \\
& +\sum_{n=0}^{N-1} \bar{x}_{m} e^{j 2 \pi m n / N}
\end{aligned}
$$

where

$$
\begin{aligned}
& \sum_{n=0}^{N-1} e^{j 2 \pi m n / N}=N \\
& \hat{x}_{m}^{\prime}=x_{m}+N \log A ; \quad m=0 \\
& =x_{m} ; \quad m=1,2,3, \ldots, N-1 . .
\end{aligned}
$$

\section{5 Shifting}

The input waveform is now shifted in time to investigate how that affects the computed complex cepstrum. A mathematical development shows the first computed,cepstral points of both the shifted and nonshifted input waveforms are identical. The remainder of the shifted cepstral points are modified but functionally related to the nonshifted cepstral puints.

$$
\text { Let } \begin{aligned}
x_{m} & =x_{m-k} \\
\bar{x}_{m}^{\prime} & =\operatorname{DFT}\left[x_{m}^{\prime}\right]=\bar{x}_{m} e^{-j k \omega_{m} T},
\end{aligned}
$$


where

$$
\begin{aligned}
& \omega_{\mathrm{m}}=\frac{2 \mathrm{~mm}}{\mathrm{NT}} \quad \omega_{\mathrm{m}}=0,1, \ldots, \frac{\mathrm{N}-1}{2} \\
& \frac{2 \pi(n-N)}{N T} \omega_{m}=\frac{N+1}{2}, \ldots, N-1 \\
& \log \left[\bar{x}_{m}^{\prime}\right]=\log \left|\bar{x}_{m}\right| \cdot-j k \omega_{m} \\
& \hat{x}_{m}^{\prime}=\operatorname{IDFT}\left[\log \left[\bar{x}_{m}^{\prime}\right]\right]=\frac{1}{N} \cdot \sum_{n=0}^{N \div 1} \log \left[\bar{x}_{m}^{\prime}\right] e^{j \omega_{m^{n}}} .
\end{aligned}
$$

After substitution for $\omega_{m}$ and the closed form is found, we have

$$
\hat{x}_{m}^{\prime}=x_{m}-j \frac{2 \pi k}{N}\left[\frac{e^{j \pi m} e^{j \pi m / N}}{e^{j 2 \pi m / N}}\right]
$$

where

$$
\begin{gathered}
e^{j \pi m}=\cos (m \pi)=(-1)^{m} \\
\frac{e^{j \pi m}}{\frac{e^{j 2 \pi m}}{N}-1}=-j \frac{1}{\sin \frac{m \pi}{N}}
\end{gathered}
$$

Thus

$$
\begin{aligned}
& \hat{\mathbf{x}}_{\mathrm{m}}^{\prime}=0 \quad \mathrm{~m}=0 . \\
& \hat{\mathrm{x}}_{\mathrm{m}}^{\prime}=\dot{x}_{\mathrm{m}}+\frac{\mathrm{mk}}{\mathrm{N}} \frac{(-1)^{\mathrm{m} 11}}{\sin \frac{\mathrm{m \pi}}{N}} \mathrm{~m}=1,2,3, \ldots \mathrm{N}-1 .
\end{aligned}
$$

For small angles, the sine approximation is made so that

$$
\hat{\mathbf{x}}_{\mathrm{m}}^{\prime}=\mathrm{x}_{\mathrm{m}}+\frac{\mathrm{k}}{\mathrm{m}}(-1)^{\mathrm{m}} \mathrm{m}=1,2,3, \ldots, \mathrm{N}-1
$$

Recalling from Section 3.2, an input normalization requirement is to reposition the waveform to the origin by the amount of lag. To illustrate the shifting phenomenon, this procedure is disregarded. 


\section{6 Other Aspects}

This section dis isses two additional aspects of the cepstrum, The first is that, for a real causal sequence, the c implex cepstrum can be computed from only the real part of the DFT. The second aspect is a redcvelopment of a mathematical relationship between the input sequence and the output cepstrum.

3.6. 1 Causality -- Fourier transform techniques are used herein for mathematical analysis. Thus, there exists mathematical relationships between the real and imaginary parts or the magnitude and phase of the Fourier transform. These relationships are called Hilbert transform relationships.

The investigated sequence must be real, causal, and stable. The intent of this section is to derive relationship between the real and imaginary parts of the Fourier transform of that sequence. This Hilbert relationship will allow the DFT of a real, causal, and stable sequence to be defined by only the real or imaginary part. Thus, the complex cepstrum can be computed using only the real portion of the DFT.

Let the sequence be expressed as the sum of an even and an odd sequence,

$$
s(n)=s_{e}(n)+s_{0}(n)
$$

where

$$
\begin{aligned}
& s_{e}(n)=1 / 2[s(n)+s(-n)] \\
& s_{o}(n)=1 / 2[s(n)-s(-n)] .
\end{aligned}
$$

Since the sequence is causal, $s(n)$ can be completely recovered from $s e^{(n) \text {. }}$

$$
s(n)=s e^{(n) \bar{s}(n)}
$$

where

$$
\vec{s}(n)=\begin{aligned}
& 2, n<0 \\
& 1, n=0 \\
& 0, n>0
\end{aligned} .
$$

The Fourier transform is

$$
S(j \omega)=S_{R}(j \omega)+j S_{l}(j \omega)
$$

and $S_{R}(j \omega)$ is the Fourier transform of $s_{e}(n)$. Then using $E_{q}(39), s(n)$ is defined. 
In terms of the Z-transform, $S(z)$ can be determined. any place in the unit circle from a knowledge of $S_{R}(j \omega)$ and $S(0)$. The real, stable, and causal sequence, $s(n)$, has a $Z$-transform that is analytic outside the circle. This is the region of convergence.

Thus, $x(z)$ is outside the unit circle $\left(z=r e^{j \omega T}\right.$ with $\left.r>1\right)$. Then

$$
\begin{aligned}
\left.H(Z)\right|_{z}=r e^{j \omega T} & =H\left(r e^{j \omega T}\right) \\
& =\sum_{n=0}^{N-1} s(n) r^{-n} e^{-j \omega n T}
\end{aligned}
$$

or, using $\operatorname{Eq}(40)$

$$
H\left(r e^{j \omega}=\sum_{n=-N}^{N} g(n)-s(n) r^{-n} e^{j \omega n T}\right.
$$

It is recognized that $\mathrm{H}\left(\mathrm{e}^{\mathrm{j} \omega}\right)$ is in convolutional form. $\mathrm{H}\left(\mathrm{e}^{\mathrm{j} \omega}\right)$ can be obtained as the convolution of the Fourier transform of $s_{e}(n)$ with the Fourier transform of $g(n) r^{-n}$.

$$
\begin{aligned}
& F T\left[S_{e}(n)\right]=S_{R}\left(e^{j \omega T}\right) \\
& F T\left[\bar{s}(n) r^{-n}\right]=\frac{1+r^{-1} e^{-j \omega T}}{1-r^{-1} e^{-j \omega T}} r>1 .
\end{aligned}
$$

The complex convolution theorem states:

if

$$
g(n)=x(n)^{*} h(n)
$$

then

$$
Y(z)=\frac{1}{2 \pi j}, \oint x\left(\frac{z}{v}\right) Y(v) r^{-1} d v
$$

where $\mathrm{v}$ is the complex variable of integration and the path is taken counterclockwise around the unit. rirrele.

Substitution into Eq (47) yields

$$
S(s)=\frac{1}{2 \pi j} \int \frac{S \frac{z e^{j \omega T}}{R v}\left(1-r^{-1} e^{-j \omega T}\right)}{1-r^{-1} e^{-j \omega T}} v^{-1} d v
$$


where

$$
z=e^{j \omega T}
$$

This relationsinip allows the Z-transform to be completely specified by the real part of the Fourier transform sequence. The complex cepstrum cannot be computed from the real portion of DFT $[s(n)]$.

\subsubsection{Input-Output Cepstral Relationship -- This section shows a mathematical relationship} developed between the input sequence and the complex cepstrum. The input sequence is assumed to satisfy normalization requirements. It is also taken to be composed of two components that have been convolved.' Given a discrete convolution sequence,

$$
\begin{aligned}
& x(m)=\sum_{n=0}^{N-1} x_{1}(n) x_{2}(m-n) \\
& x(m)=x_{1}(m) * x_{2}(m) .
\end{aligned}
$$

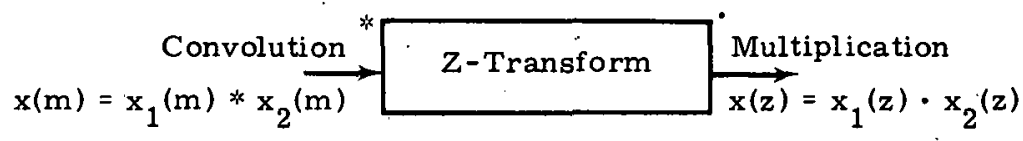

$$
\begin{aligned}
& \hat{X}(z)=\log [X(z)] \\
& =\log \left[x_{1}(z)\right]+\log \left[x_{2}(z)\right] . \\
& X(z)=\sum_{n=0}^{N-1} x(n) z^{-n} \\
& \frac{\mathrm{dX}(z)}{\mathrm{dx}} \cdot \frac{\mathrm{d}}{\mathrm{dx}} \log [X(z)]=\frac{1}{\mathrm{X}(z)} \frac{\mathrm{dX}(z)}{\mathrm{dx}} \\
& \hat{X}^{\prime}(z)=\frac{X^{\prime}(z)}{X(z)} \text {. } \\
& z \hat{X}^{\prime}(z)=\sum_{n=0}^{N-1}[-n \&(n)] z^{-n}=\frac{z X^{\prime}(z)}{X(z)} \\
& z^{\prime} X(z)=z \hat{X}^{\prime}(z) X(z)
\end{aligned}
$$

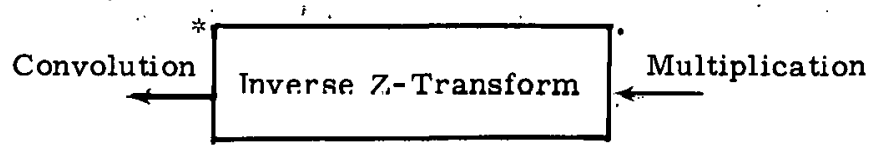




$$
\begin{aligned}
x(m)=x_{1}(m) * x_{2}(m), & x(z)=x_{1}(z): x_{2}(z) \\
z^{-1}\left[z X^{\prime}(z)\right]=m x(m) & =m \hat{x}_{1}(m) * x_{2}(m) \\
m x(m) & =\sum_{n=0}^{N-1} n \hat{x}(n) x(m-n) \\
x(m) & =\sum_{n=0}^{N-1}(n m) \hat{x}(n) x(m-n) .
\end{aligned}
$$

Thus, Eq(58) shows a recursive relationship between the input and the complex cepstrum output.

\section{Computing the Complex Cepstrum}

\section{1 Introduction}

The purpose of this section is to take a more detailed look at the restrictions and requirements involved in properly computing the complex cepstrum. The detailed computing system is depicted by Figure 8. The restrictions and requirements discussed apply to the deconvolution of seismic signals, speech, or any other data that have been recorded in a reverberant environment.

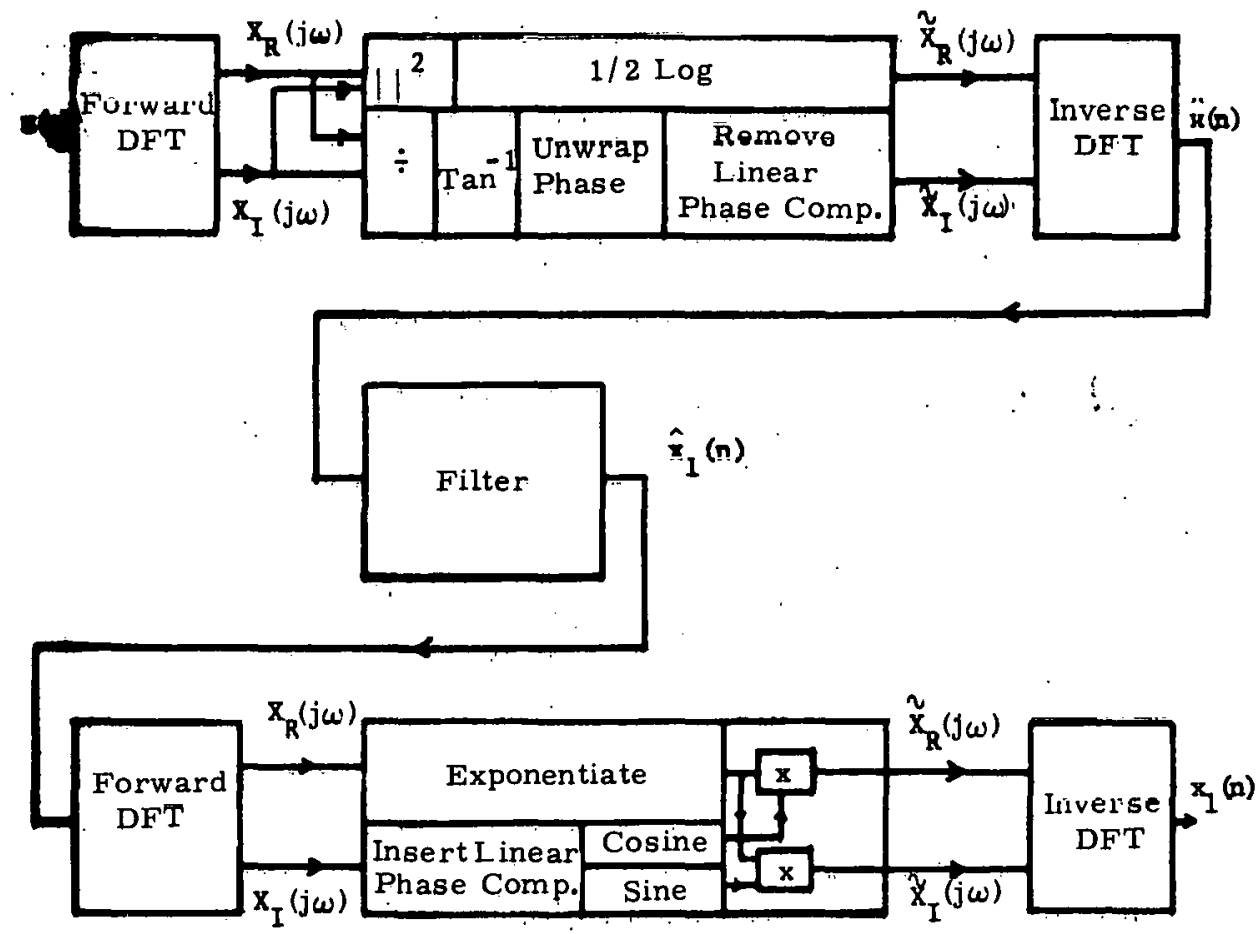

Figure 8. Detailed Homomorphic System for Deconvolution 
To fully illustrate all the computational requirements, only causal input sequences aré discussed. The complex cepstrum can be computed for maximum, minimum, and mixed-phase signals. These signals have the zeros of their rational $Z$-transforms outside, inside, and both inside and outside the unit circle, respectively.

\subsection{Input Normalization and Requirements}

The first requirement of the input sequence to the characteristic system $D_{*}$ is that the sequence be stable and have positive polarity and zero lag. If the waveform does not have positive polarity or has some lag, there are nonzero contributions added to the complex cepstrum which do not give any extra information.

Homomorphic systems for deconvolution can operate on modified maximum- and mixed-phase signals. Exponential weighting is employed to meet the minimum-phase requirements. Exponential weighting radially scales the location of the poles and zeros in the Z-plane. The exponential factor $(\alpha)$ is chosen so that the poles and zeros on the outside of the unit circle are moved sufficiently inside. The use of exponential weighting forces maximum-and mixed-phase sequences to become minimum-phase sequences. Exponential deweighting must take place after homomorphic analysis in order to restore the original characteristic for the sequences. Figure 9 depicts the weighting process.

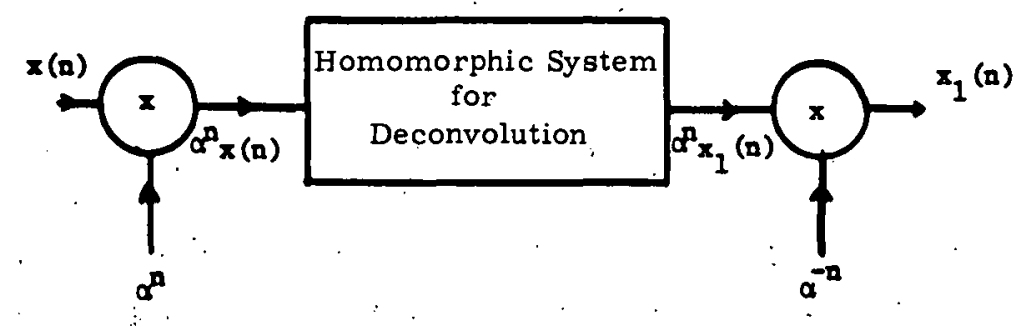

Figure 9. Homomorphic System for Öperation on a Maximumor Mixed-Phase Sequence

\section{3 Phase Unw rapping}

Phase unwrapping becomes necessary with the evaluation of the complex logarithm. Equation (42) can further be expressed as

$$
\begin{aligned}
\log \left[\bar{x}_{n-k}\right] & =\log [|\bar{x}(j \omega)| \exp [j \operatorname{Arg}(\bar{x}(j \omega))]] \\
\therefore & =\log |\bar{x}(j \omega)|+j \operatorname{Arg}[\bar{x}(j \omega)] .
\end{aligned}
$$

It is seen that the logarithm of the complex number is another complex number. 
To properly compute the complex cepstrum, the explicit inverse Fourier transform of the DFT logarithm of the input must be computed. The complex cepstrum is defined as a real sequence. Recall that the DFT of a real sequence hạs an even portion and an odd portion with the period $2 \pi / T$.

This requires that the logarithm be appropriately defined with the same periodicity. The magnitude of the DFT generally offers no uniqueness problem and satisfies this requirement.

The problem arises because the phase angle of the DFT is only unique to within an integer multiple of $2 \pi$. Usually the phase of the Fourier transform is a computed module $2 \pi$. This yields a discontinuous phase curve. A simple algorithm can be written to unwrap the phase curve based on the examination of the sampled phase to locate jumps of $2 \pi$.

An alternate and more sophisticated approach based on the work of Tribolet is an adaptive numerical integration of the derivative of the phase function. ${ }^{7}$ The derivative of the phase portion is shown below:

$$
\begin{aligned}
\operatorname{Arg}^{\prime}[\bar{X}(j \omega)] & =\frac{d \operatorname{Arg}[\bar{X}(j \omega)]}{d \omega} \\
& =\frac{x_{R}^{(j \omega) X_{I}^{\prime}(j \omega)-X_{I}(j \omega) X_{R}^{\prime}(j \omega)}}{x_{R}^{2}(j \omega)+x_{I}^{2}(j \omega)},
\end{aligned}
$$

where

$$
\bar{x}^{\prime}(j \omega)=x_{R}^{\prime}(j \omega) \text { I j } x_{I}^{\prime}(j \omega)
$$

is computed as - j DFT $[n x(n)]$. Based on Rienmann surface theory, integration of the derivative of the log function gives a continuous plane for its evaluation. The adaptive numerical integration scheme is applied with initial condition $\omega=0$.

$$
\operatorname{Arg}[\bar{x}(j \omega)]=\int_{0}^{\omega} \operatorname{Arg}[x(j \omega)] d \omega,
$$

where initial condition $\operatorname{Arg}[X(0)]=0$.

This technique of phase unwrapping ensures that the imaginary nnsition is nnnamhigusus, odd, and a periodic function of as.

Consider the complex cepstrum to be computed for the waveform shown in Figure 10 . Without using a phase-unwrapping technique, the phase curve is computed using the basic intrinsic arc tangent routine. 


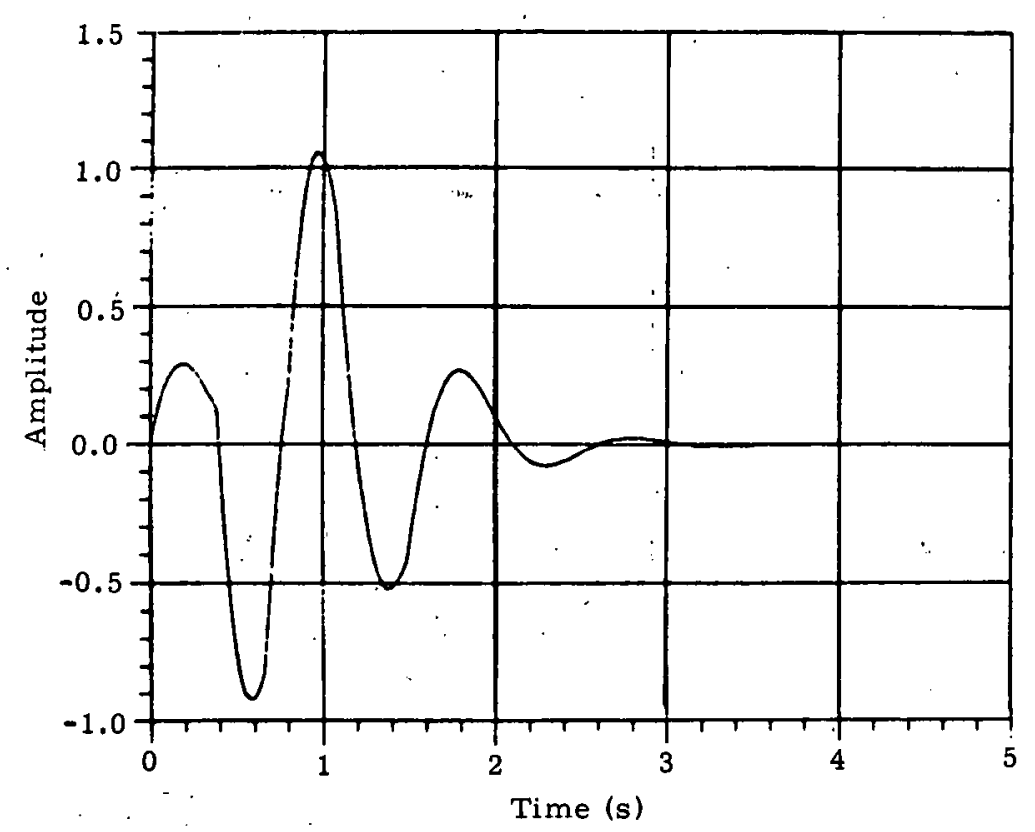

Figure 10. Input Waveform to the Homomorphic System

Figure 11 shows the discontinuous nature of the principal value of the phase.

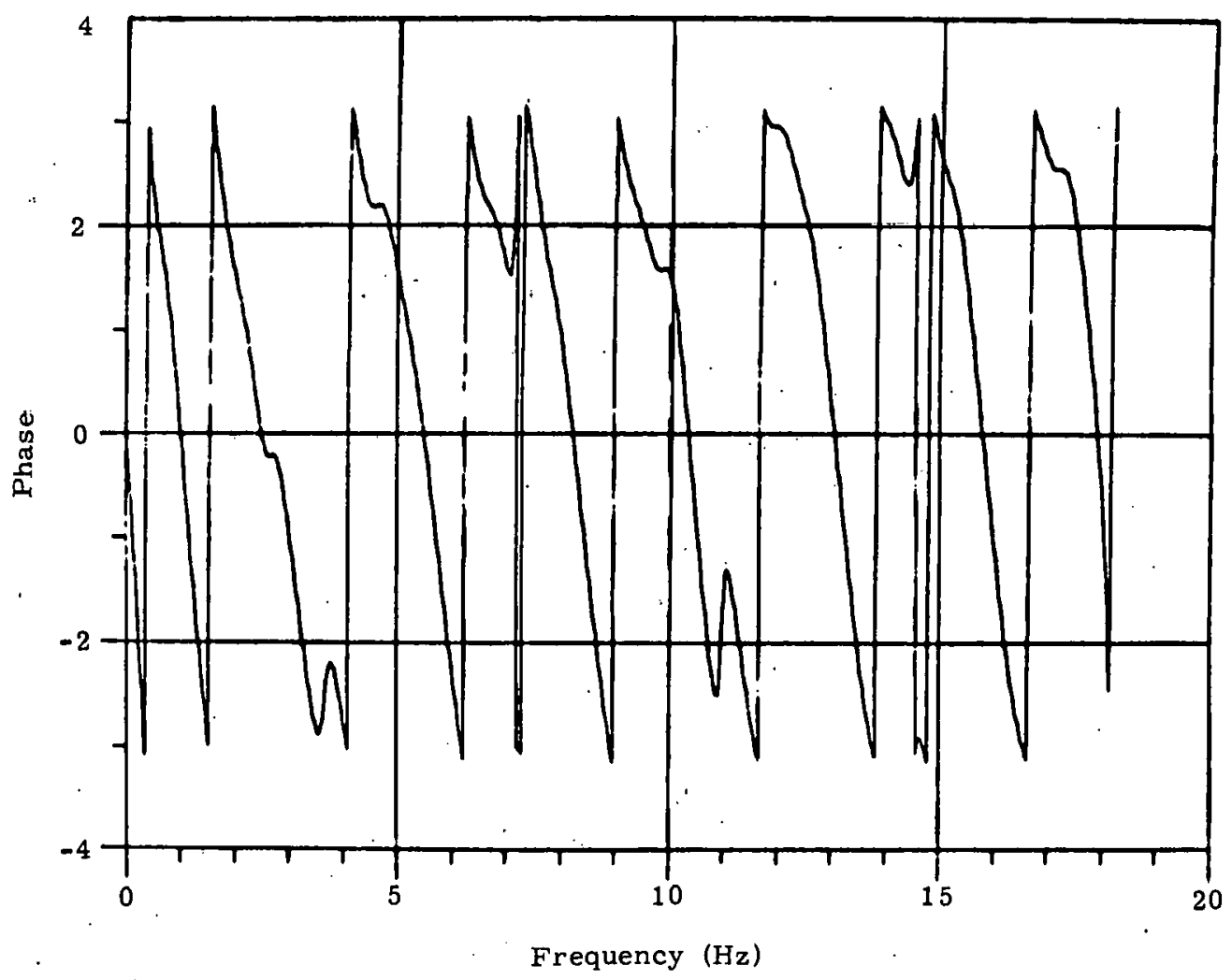

Figure 11. Principal Value of the Phase 
Proper computation of the complex cepstrum requires a continuous phase curve. Adaptive numerical integration of the phase derivative gives a continuous phase curve and is shown in Figure 12.

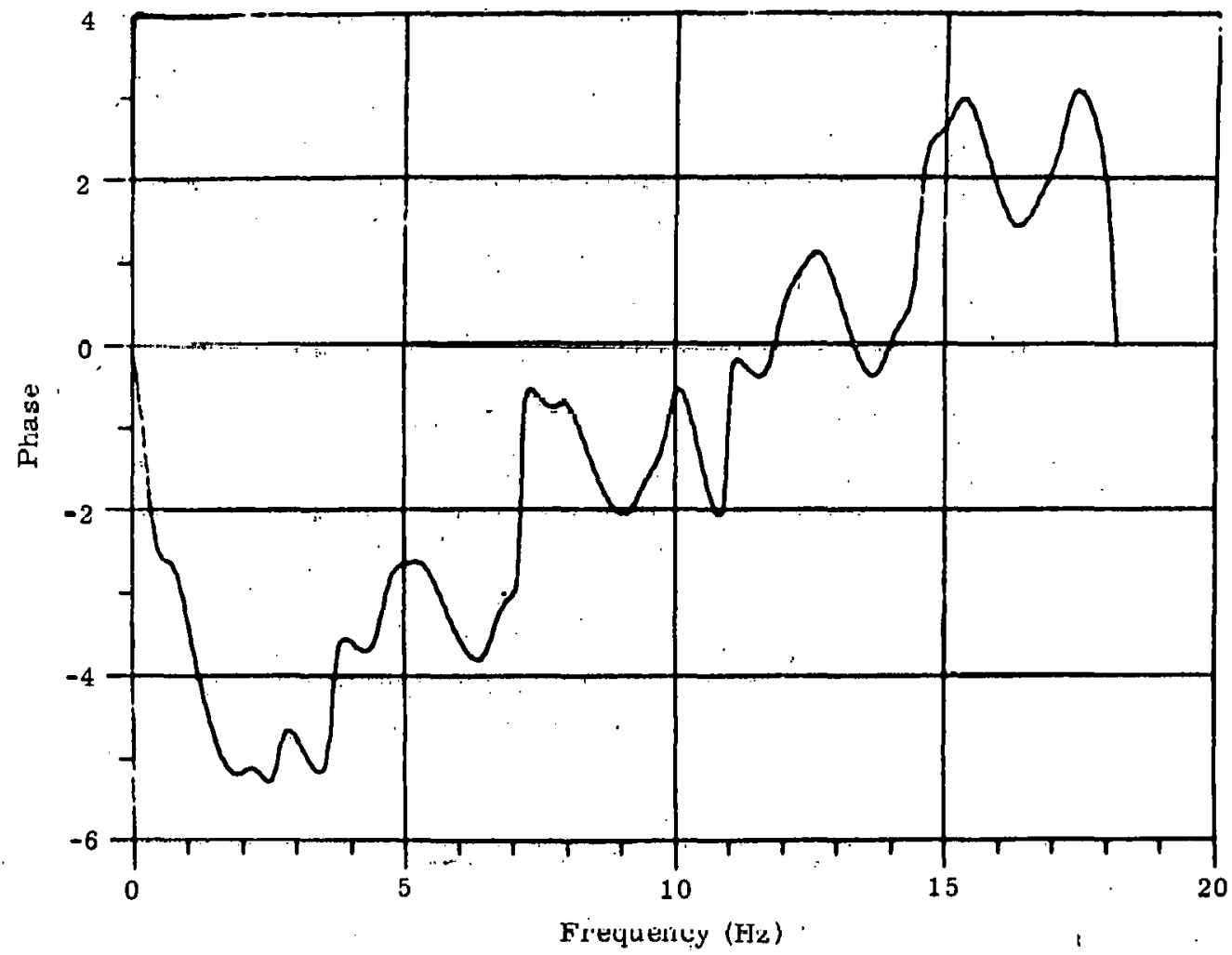

Figure 12. The Unwrapped Phase With the Linear-Phase Component Hemoved

\subsection{The Linear-Phase Component}

The linear-phase component appears as a result of implementing the numerical integration technique. It is introduced by taking the derivative of the log phase term, Eq (60). The integral of the derivative is substituted back into the complex Fourier-transformed sequence which contains a linear-phase component in the frequency dọmain.

A scheme is devised to keep track of the numerical value of the linear-phase component as the unwrapped-phase curve is calculated. After phase unwrapping, the numerical value of the linearphäsé component can be removed.

A linear-phase component in the frequency domain implies a shift in the time domain. This gives another option for its removal. If the linear component is included and transformed back to the time domain, it can be removed by simply repositioning the waveform. 


\section{Deconvolution Using a Known Waveform}

\subsection{Introduction}

The purpose of this section is to deconvolve a composite waveform that has been made up of two known components. This task was undertaken to test the computer codes and determine how well this convolution technique works.

\section{2 Fabrication of the Input}

The input waveform to the homomorphic system is made up of a simple decaying sine wave convolved with a sequence of two impulses. The convolutional components are shown in Figures 13 and 14, respectively; the final convolutional results are shown in Figure 15.

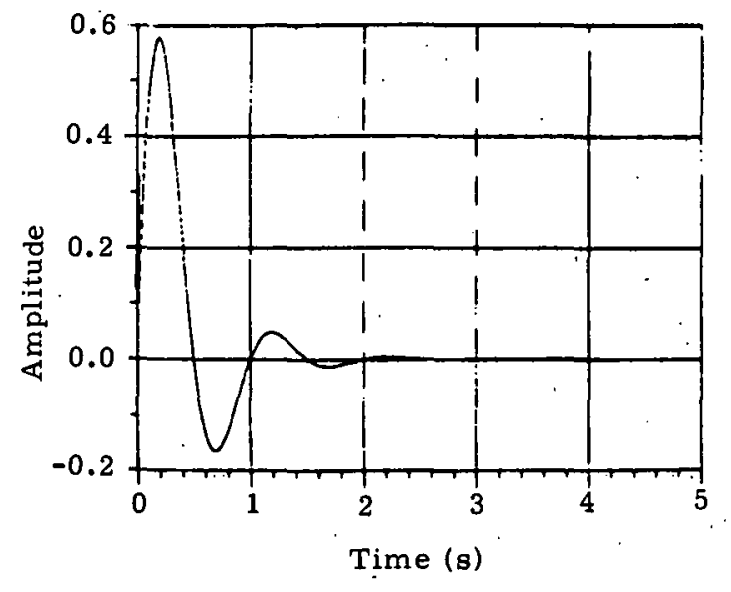

Figure 13. Decaying Sine Wave

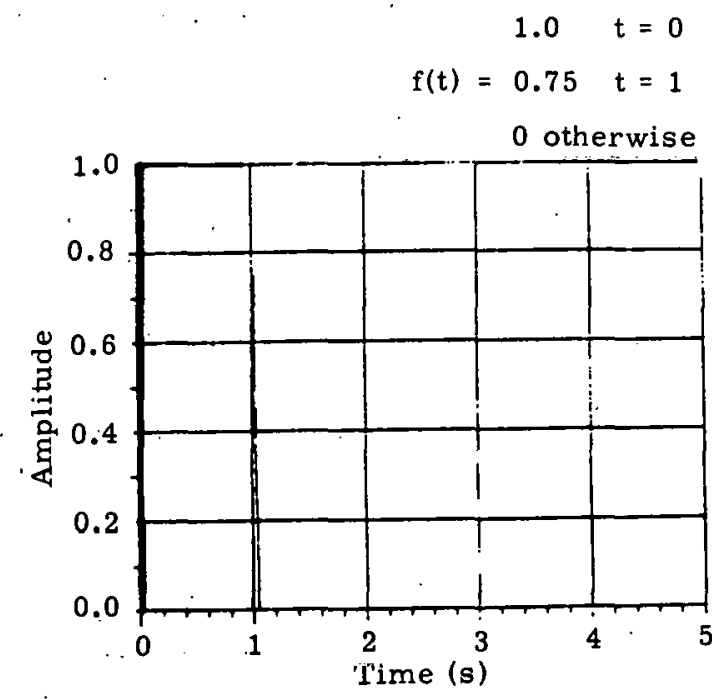

Figure 14. Sequence of Two Impulses

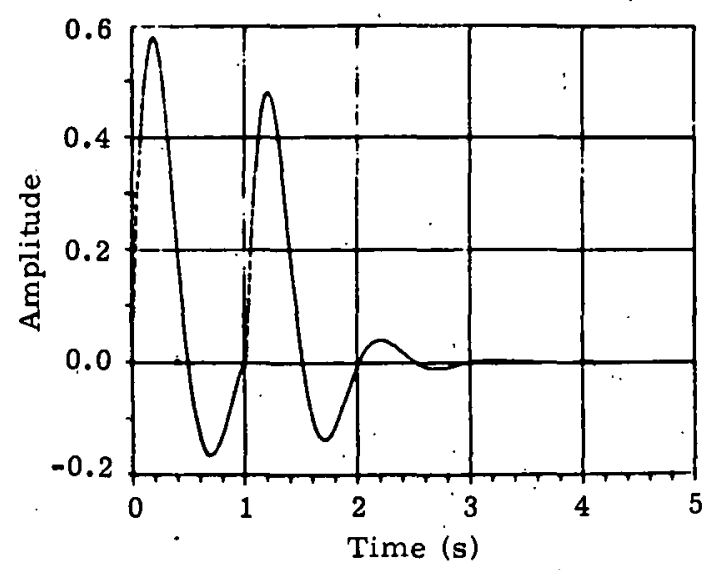

Figure 15. Convolutional Rcoult 
The convolution of the two known components was performed by taking the product of their DFTs and inverse Fourier tranșforming.

\section{3 The Computed Complex Cepstrum}

Since the waveform under consideration is minimum-phase, its rational $Z$-transform has no poles or zeros outside the unit circle. This allows the cepstrum to be computed using adaptive numerical integration of the phase derivative.

Two independent computer codes were written for deconvolution. The first is more elaborate and is for operation on minimum-phase signals only. This program employs a phase-unwrapping algorithm written by Tribolet. ${ }^{10}$ Modifications were made to the algorithm that made it workable with the Sandia FFT routines and the Sandia general-purpose digital computer. The algorithm takes care of any normalizations required and removes the linear-phase component induced by taking the derivative. This code cannot unwrap the phase curve of maximum- and mixed-phase waveforms.

The second code is a much simpler program and can operate on minimum-, maximum-, and mixed-phase waveforms. A more general and less complex unwrapping technique is used. Advanced phase-unwrapping techniques are not applicable to maximum- and mixed-phase waveforms. This program requires that normalization requirements be satisfied before this phase-unwrapping procedure. This method is preferred for application of seismic records since they are, in general, mixed-phase.

The advanced phase-unwrapping technique will be used in computing the complex cepstrum of the real and causal fabricated waveform in Figure 15. The first, more elaborate code will work successfully provided the waveform is minimum-phase. The waveform turns out to be minimumphase and the computed complex cepstrum is shown in Figure 16.

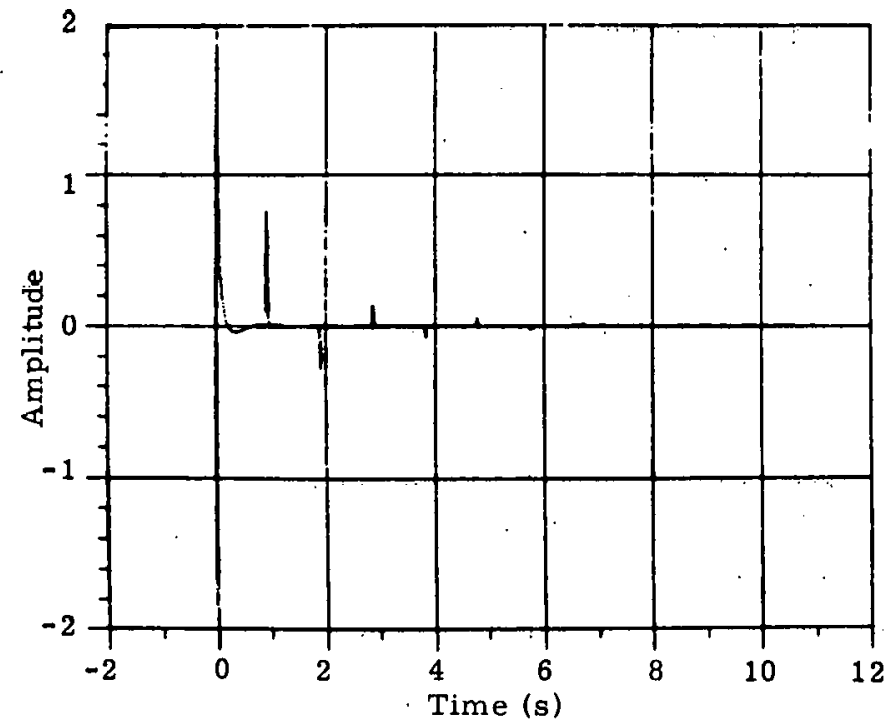

Figure 16. Computed Cumplex Cepslrum 


\section{4 Linear Filtering}

Cepstrum theory states that the slowly varying convolutional component will tend to be concentrated about the origin in the cepstral domain. The more rapidly varying component will tend to occupy the remainder of the cepstral domain. For seismic signals the source component will vary much more slowly than the impulse response component.

It is known that the complex cepstrum of a sequence of impulses will also be a sequence of impulses. With this knowledge and the fact that the convolutional space is now an additive space, a low-time gate of $0.98 \mathrm{~s}$ was used on the computed complex cepstrum shown in Figure 16. All information up to $0.98 \mathrm{~s}$ is kept and shown in Figure 17; this is labeled as the low-time cepstral component. The remainder of the information is also of interest and shown in Figure 18, labeled as the high-time component.

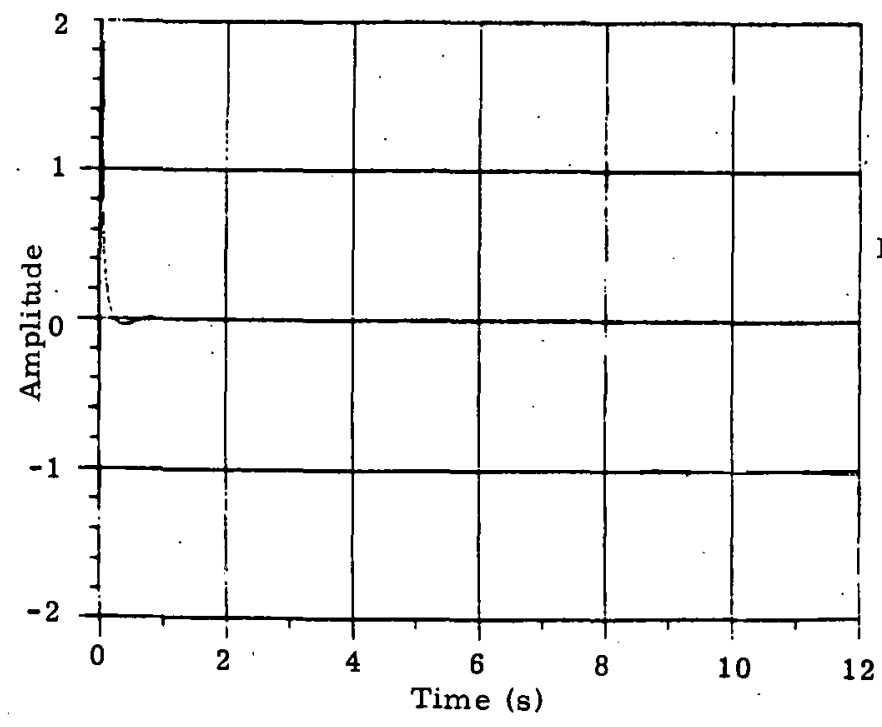

Figure 17. Low-Time Cepstral Component

Figure 18. High-Time Cepstral Component

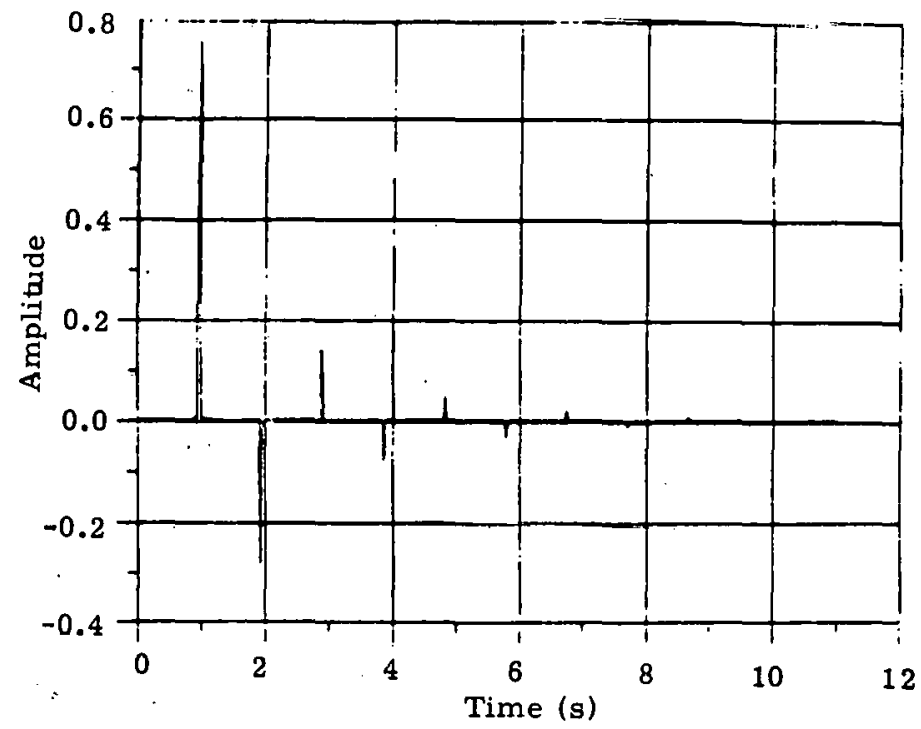




\section{5 Results}

The final step and test is to reverse the operations used to compute the complex cepstrum. This is performed by the inverse characteristic system $\mathrm{D}_{*}^{-1}$ with the low- and high-time cepstral components as separate inputs.

The final results are shown in Figures 19 and 20, respectively. Comparison with Figures 13 and 14 shows that they are the original convolutional components; a small error has been introduced which can be attributed to the round-off errors of the computing process.

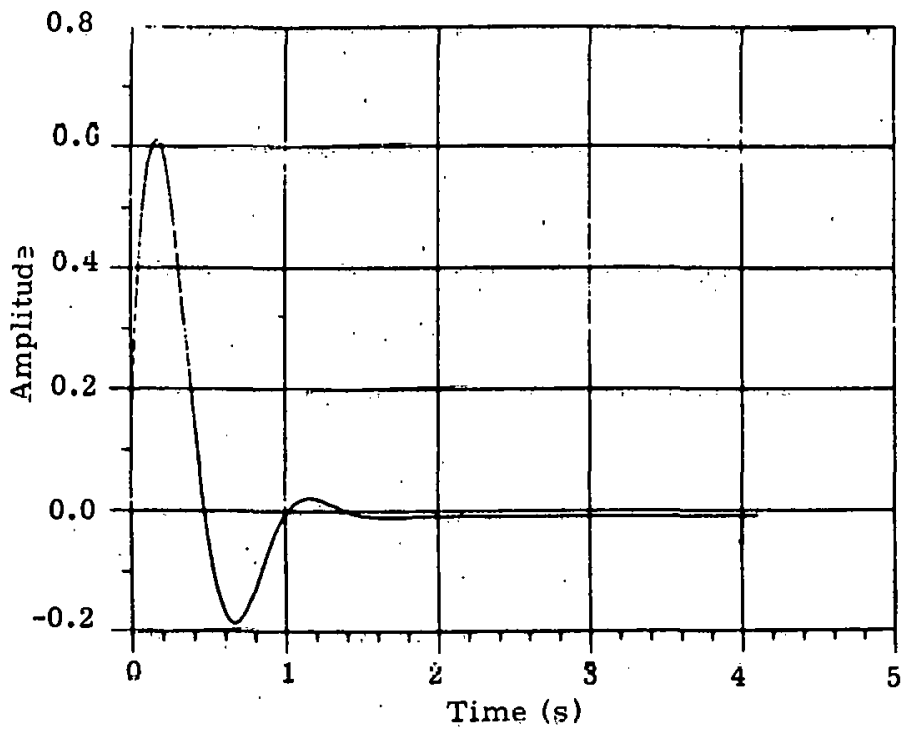

Figure 18. Reconstructed Waveform for the Low-Time Component

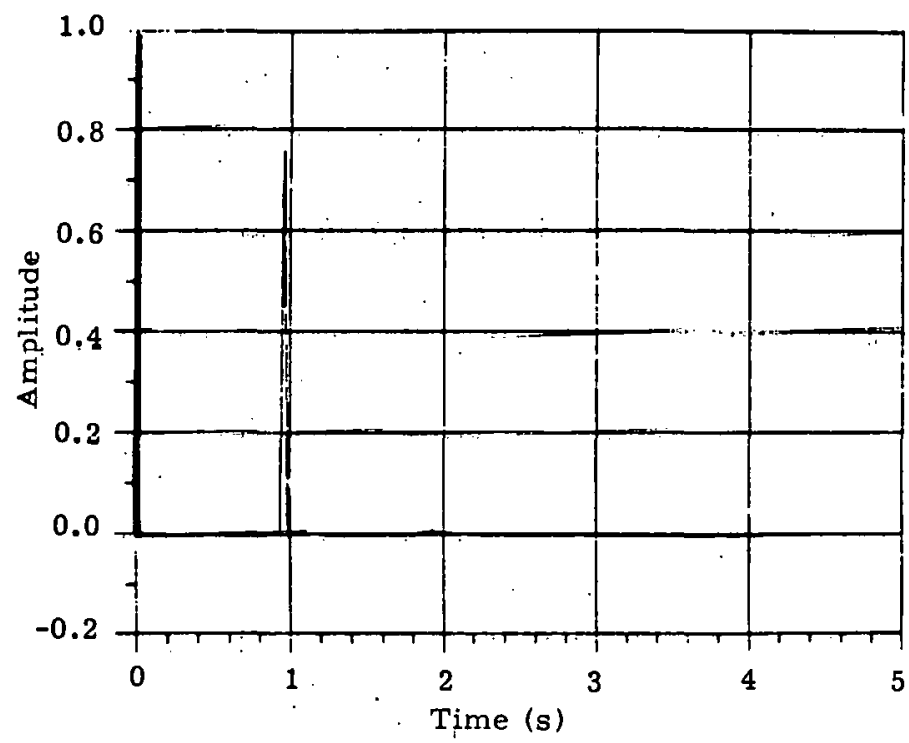

Figure 20. Reconstructed Waveform for the High-Time Component 


\section{A Computation With Real Data}

\section{1 Introduction and Background}

At this point a generalized linear filtering system is applied to "real-world" seismic data. The task is to deconvolve the seismic trace into two separate components. Specifically one component describing the physical process and the other component describing the impulse response of the medium traveled.

A typical seismic trace (see Figure 21) was chosen for investigation. The trace was sampled to avoid aliasing at 50 samples per second. The time duration of the trace is $\sim 40 \mathrm{~s}$.

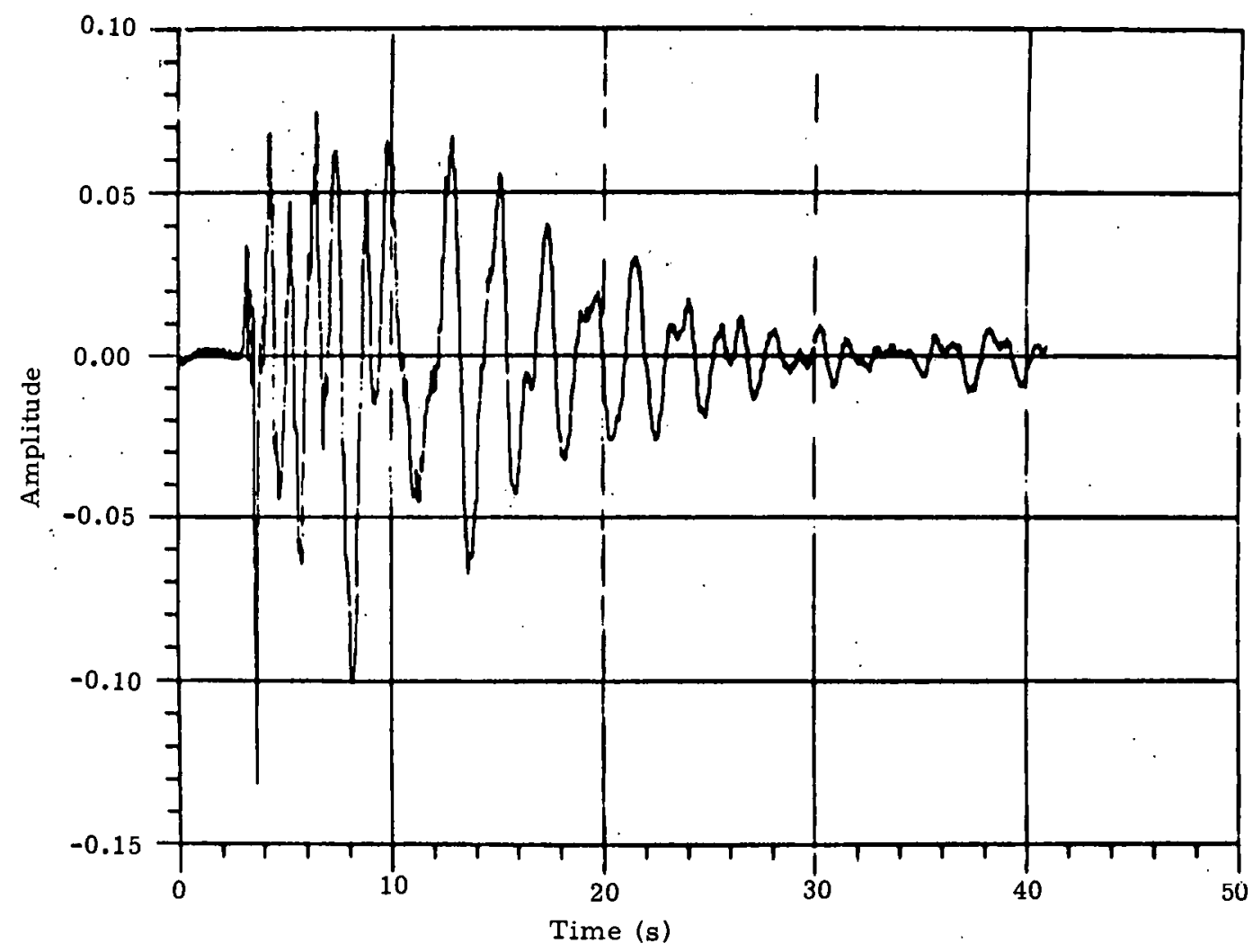

Figure 21. Unfiltered Seismic Signal (courtesy of Sandia Laboratories)

\section{2 Procedure of Computation}

The initial step is to compute the power spectrum of the waveform. Seismic signals are usually devoid of high-frequency content. It is desirable to determine a cutoff frequency from the power spectrum. The cutoff frequency is chosen at the point of negligible power with respect to the entire spectrum. 
Consider the typical seismic waveform in Figure 21. The power spectrum is computed and shown in Figure 22. From inspection and engineering judgment, the cutoff frequency picked was $20 \mathrm{~Hz}$.

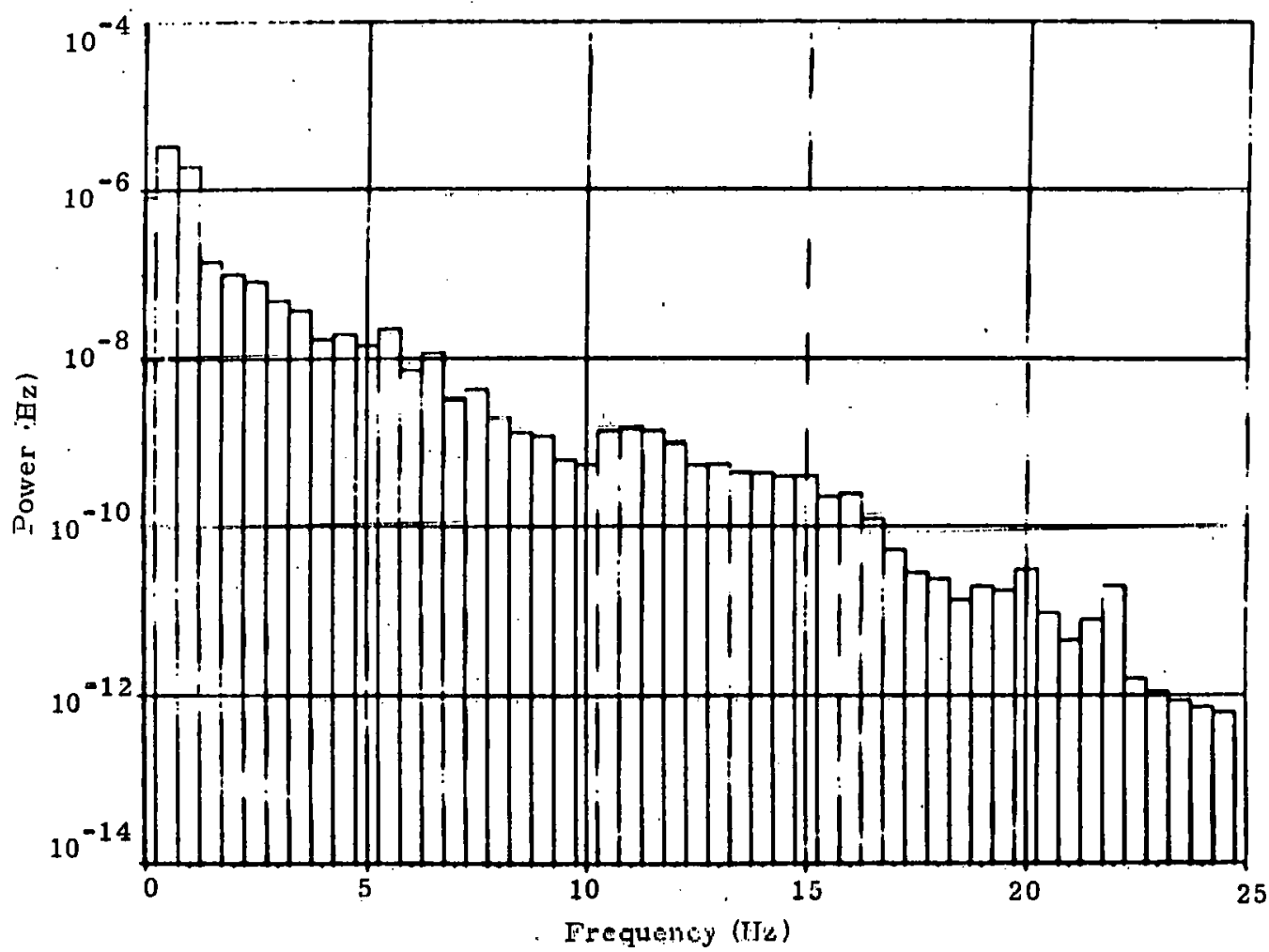

Figure 22. The Power Spectrum at the Seismic Input

This information is used to design a low-pagg filter. The waveform is filtercd so that the frequency content of the initial data is known.

A low-pass Butterworth-type filter of eight sections was designed with a cutoff of $20 \mathrm{~Hz}$ and a transition band of $2 \mathrm{~Hz}$ with an attenuation of $140 \mathrm{~dB}$. A plot of the filter gain is shown in Figure 23. No information is to be altered or lost below the cutoff frequency of the wayeform; therefore there cannot be a phase shift as a result of the filtering.

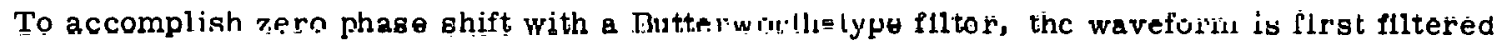
forward and then refiltered, reversed in time; the results are shown in Figure 24. The next step is to satisfy the normalization requirements and filter with the homomorphic system.

This seiemic wavcform is cunsidered to be mixed-phase. A computer program was written employing the simple phase-unwrapping technique used for the analysis (recall separate descriptions, Section 5. 3). This was necessary because the complex cepstrum has contributions in both positive and negative time. The program as written does not require the use of exponential weighting. The complex cepstrum was computed and is shown in Figure 25. 


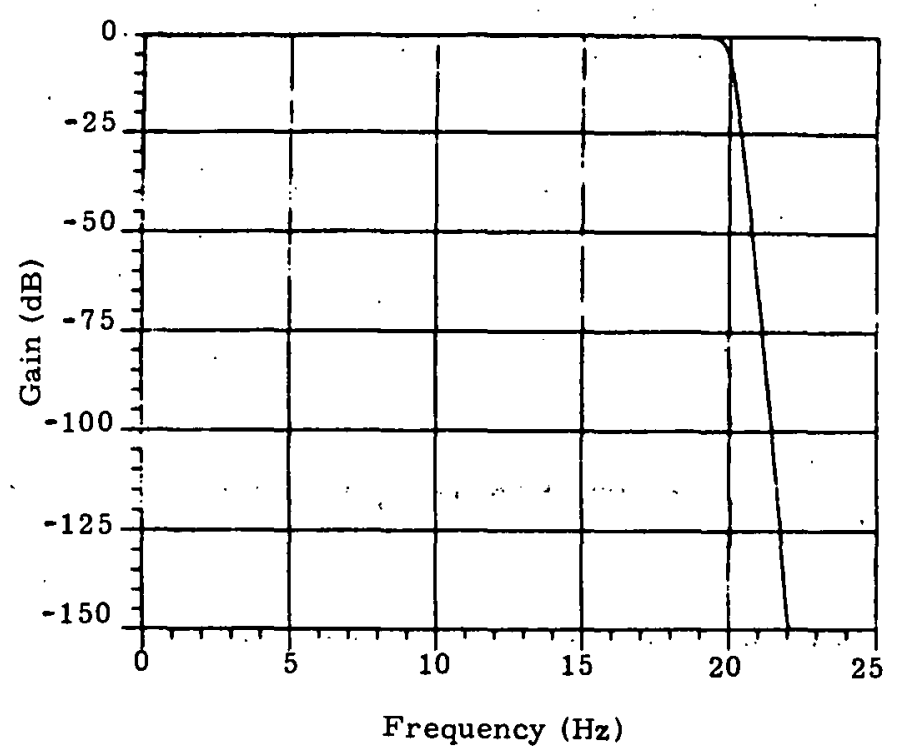

Figure 23. Low-Pass Butterworth Type Filter (8 sections)

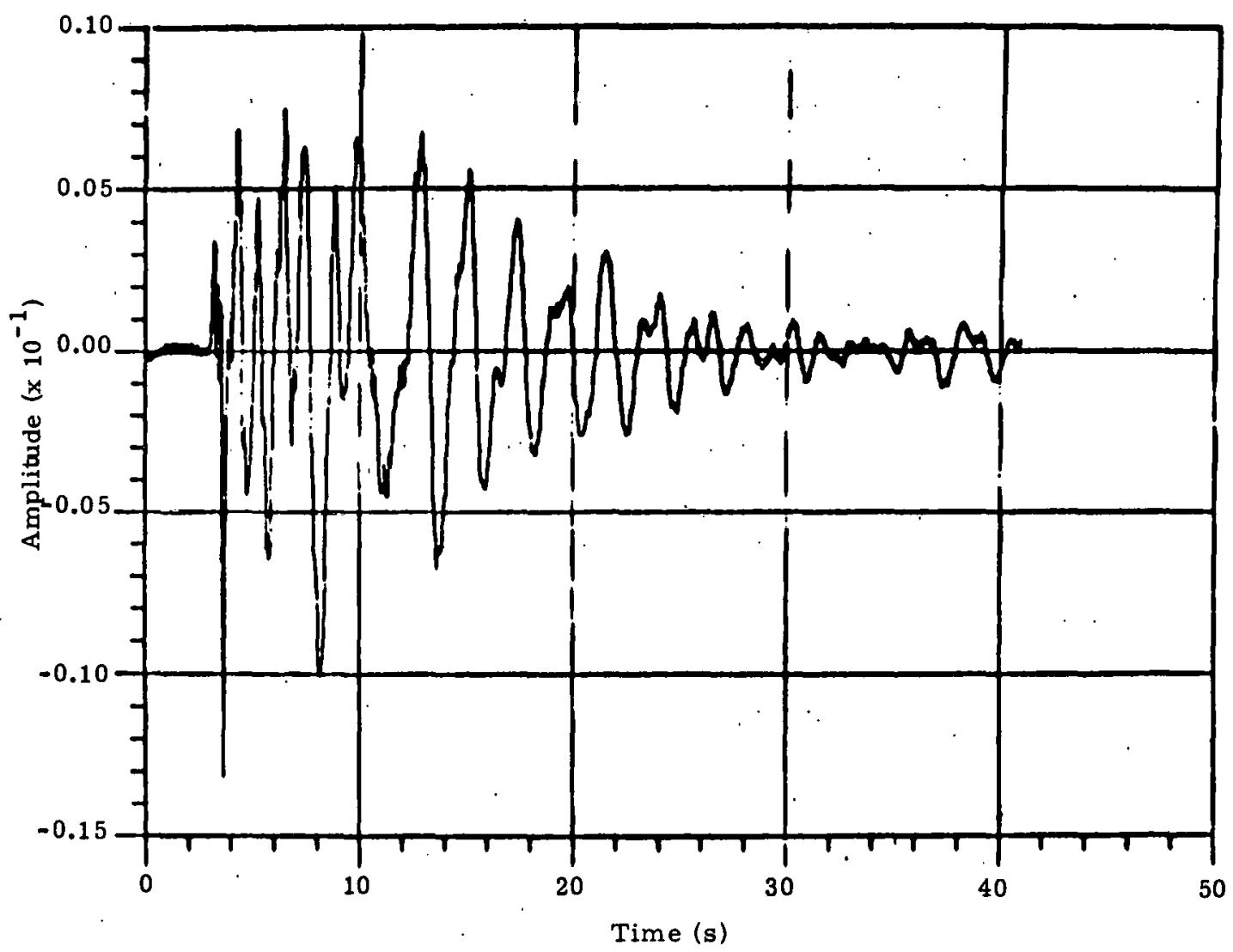

Figure 24. Low-Pasa Filtered Input Waveform 
Using the arguments of Section 5:4, a symmetrical low-time gate of 1.2-s duration was used to separate the low-time component from the high-time component.as shown in Figures 26 and 27. respectively.

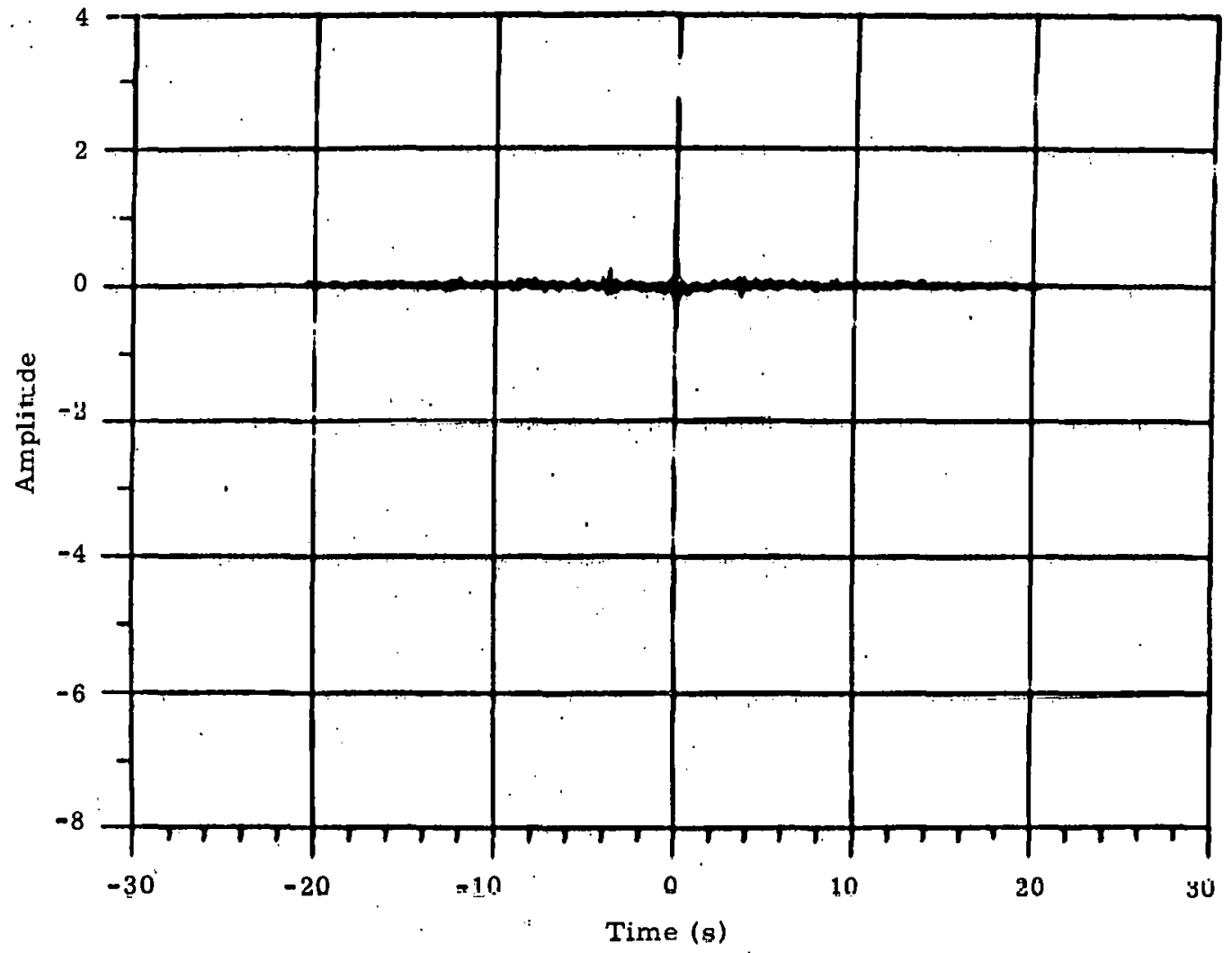

lig gule 25. Computed čomplex Censitrum

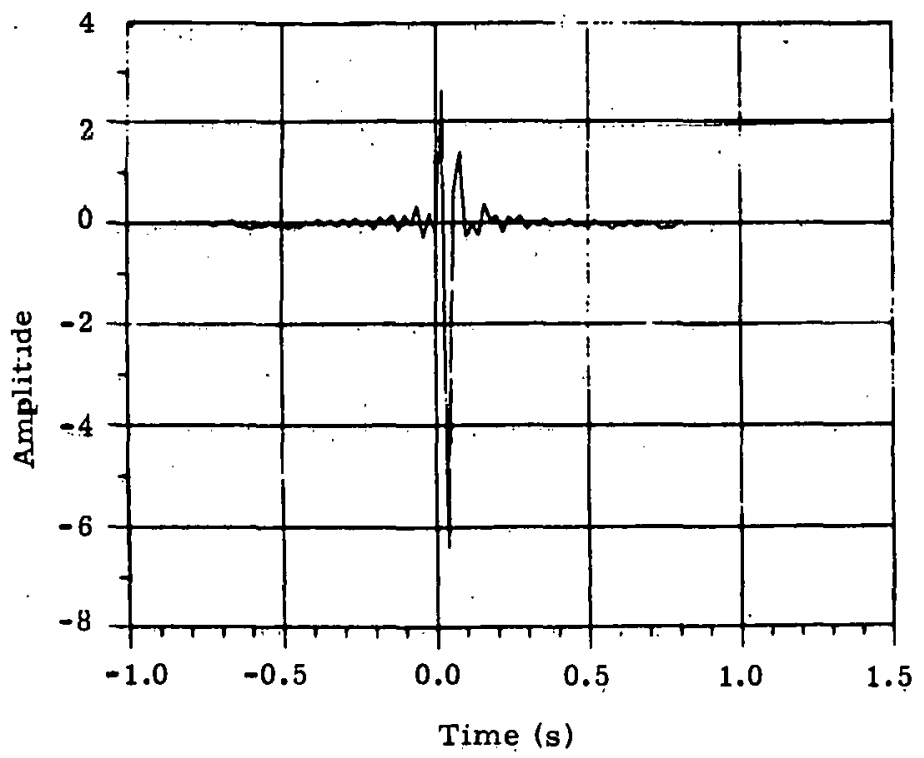

Figure 26. Low-Time Cepstral Component 


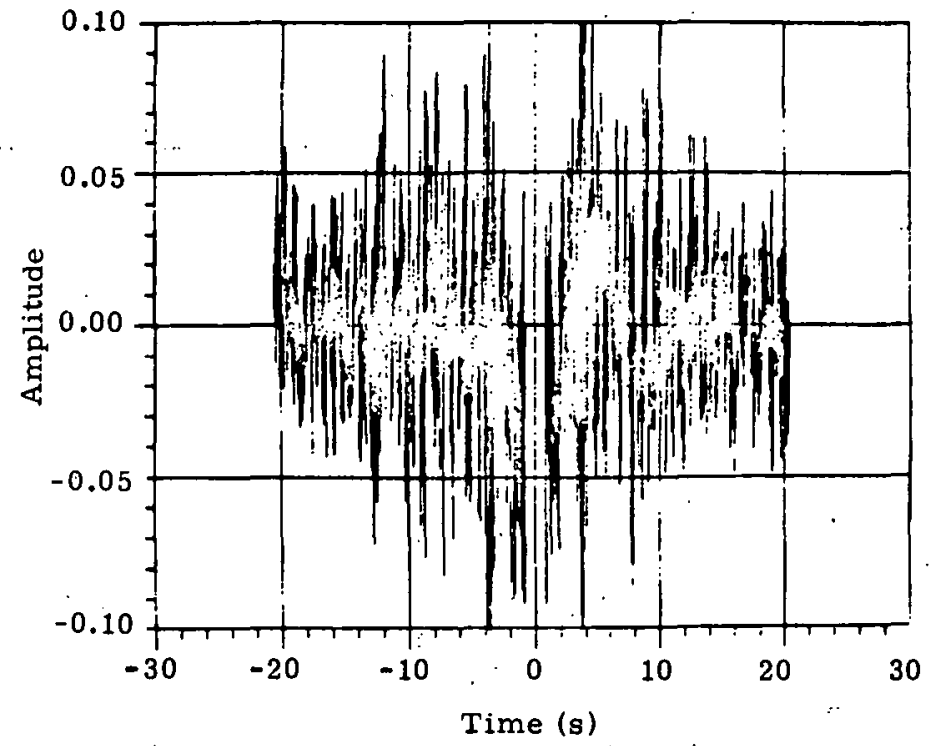

Figure 27. High-Time Cepstral Component

\subsection{Results and Conclusions}

The inverse transformation operations were performed on the low-time and the high-time components. The low-time component yields the source wavelet and is shown in Figure 28. The high-time component yields the response of the medium traveled through and is shown in Figure 29.

These two components were recovered without any prior knowledge of seismic signal composition. Thus, an analytical interpretation should be made regarding the usefulness of these results. Information $j$; available about the physical phenomenon, the recording site, and the material composition of the path traveled. The information about the location, position, and depth of the recording sensor and the theoretical characteristics of the material traveled through with respect to sound transmission are also known. This information will aid in determining the correctness of the suurce wavelet and the response of the merium traveled.

A significant amount of these calculations should be performed; the seismic records investigated should reflect a variety of conditions. The number of samples in each case to make the assessment will depend on the initial record length and the power spectrum of that record. Analysis of the results will help to determine how well the homomorphic system works for deconvolution recovery from seismic records of the source wavelet and the response of the medium traveled. 


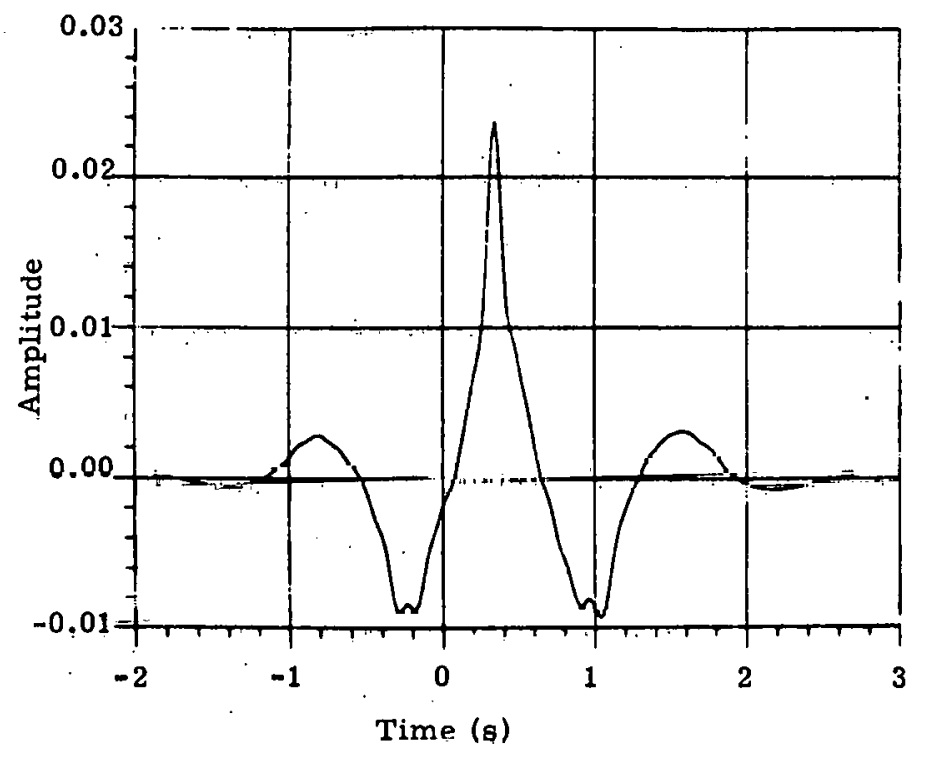

Figure 28. Computed Source Wavelet

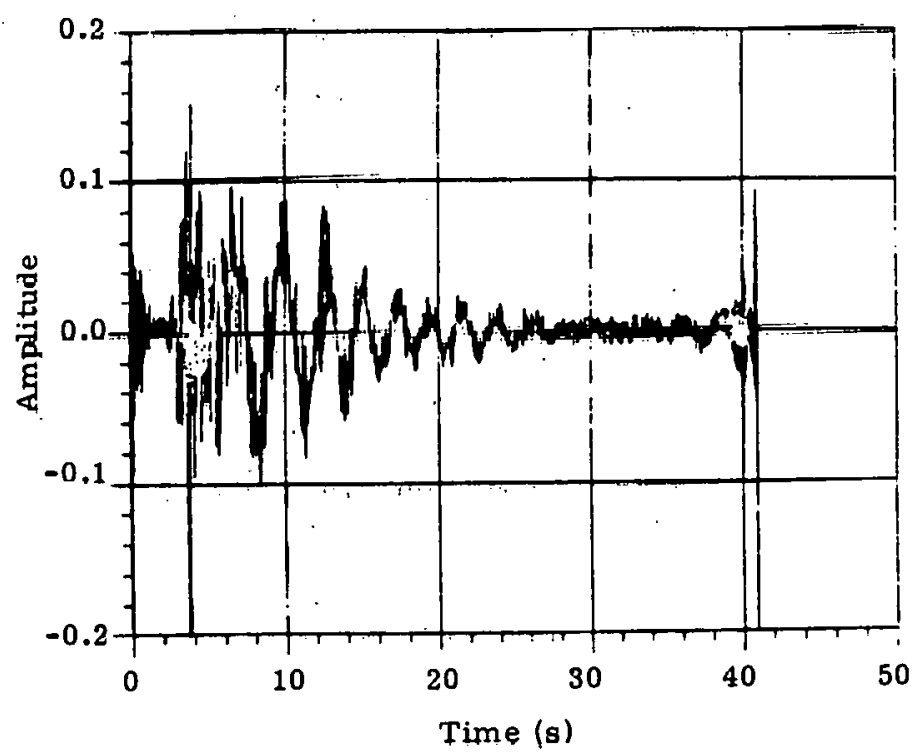

Figure 29. Computed Impulse Response of the Medium Traveled 
1. A. V. Oppenheim and R. W. Schafer, Digital Signal Processing (Englewood Cliffs, NJ: Prentice Hall, 1975) Ch 10.

2. A. V. Oppenheim, "Generalized Superposition," Information and Controi, Vol 11, Nos 5-6, November-December 1967, pp 528-536.

3. B. P. Bogert, M. J. R. Healy, and J. W. Tukey, "The Quefrency Alanysis of Time Series for Echoes: Cepstrum, Pseudo-autocovariance, Cross-Cepstrum, and Saphe Cracking, " Proc Symp Time Series Analysis, M. Rosenblatt, Ed., New York, John Wiley \& Sons, Inc, New York, 1963, pp 209-243.

4. J. M. Tribolet, Seismic Applications of Homomorphic Signal Processing (Englewood Cliffs, NJ: Prentice Hall, 1978).

5. T. Ulrych, O. G. Jensen, R. M. Ellis, and P. G. Sommerville, "Homomorphic Deconvolution of Some Teleseismic Events," Bull Sesmol Soc America, Vol 62, No 5, March 1972, pp 12531265.

6. J. M. Tribolet, T. Quatieri, and A. V. Oppenheim; "Short-Time Homomorphic Analysis," 1977 IEEE Conference on Acoust, Speech, and Signal Processing, Hartford, CT.

7. A. V. Oppenheim, Superposition in a Class of Non-Linear Systems, Tech Report 432 (Cambridge, MA: Research Laboratory of Electronics MIT, 1965).

8. N. Levinson and R. M. Redheffer, Complex Variables (Cambridge, MA, Holden-Day, Inc, 1970) pp 70-76.

9. L. V. Ahlfors, Complex Analysis (New York: McGraw-Hill, 3rd ed, 1979) pp 97-98.

10. J. M. Tribolet, "A New Phase Unwrapping Algorithm," IEEE Trans Acoust, Speech, Signal Processing, Vol ASSP-25, April 1977, pp 170-179. 
DISTRIBUTIỌN :

North Carolina A\&T University

Electrical Engineering Dept

Greensboro, NC 27411

Attn: W. Alexander

H. B. Riley (5)

204 N. Swing Rd

Greensboro, NC 27411

Dikewood Computer Corp.

1009 Bradbury Dr, SE

Albuquerque, NM 87106

Attn: T. S. Mc Donald

1110 J. D. Plimpton

1111 J. R. Banister

1111 L. R. Hịll

1111 S. D. Stearns

1111 L. J. Vortman

1 ?.R? P. A. Stokea

1262 C. D. Lundergan

4510 W. D. Weart

5642 D. E. Amos

8266 E. A. Nas

3141 T. L. Werner (5)

3151 W. L. Garner (3)

For DOE/TIC (Unlimited Release)

DOE/TIC (25)

(J. Hernandez, 3154-4) 


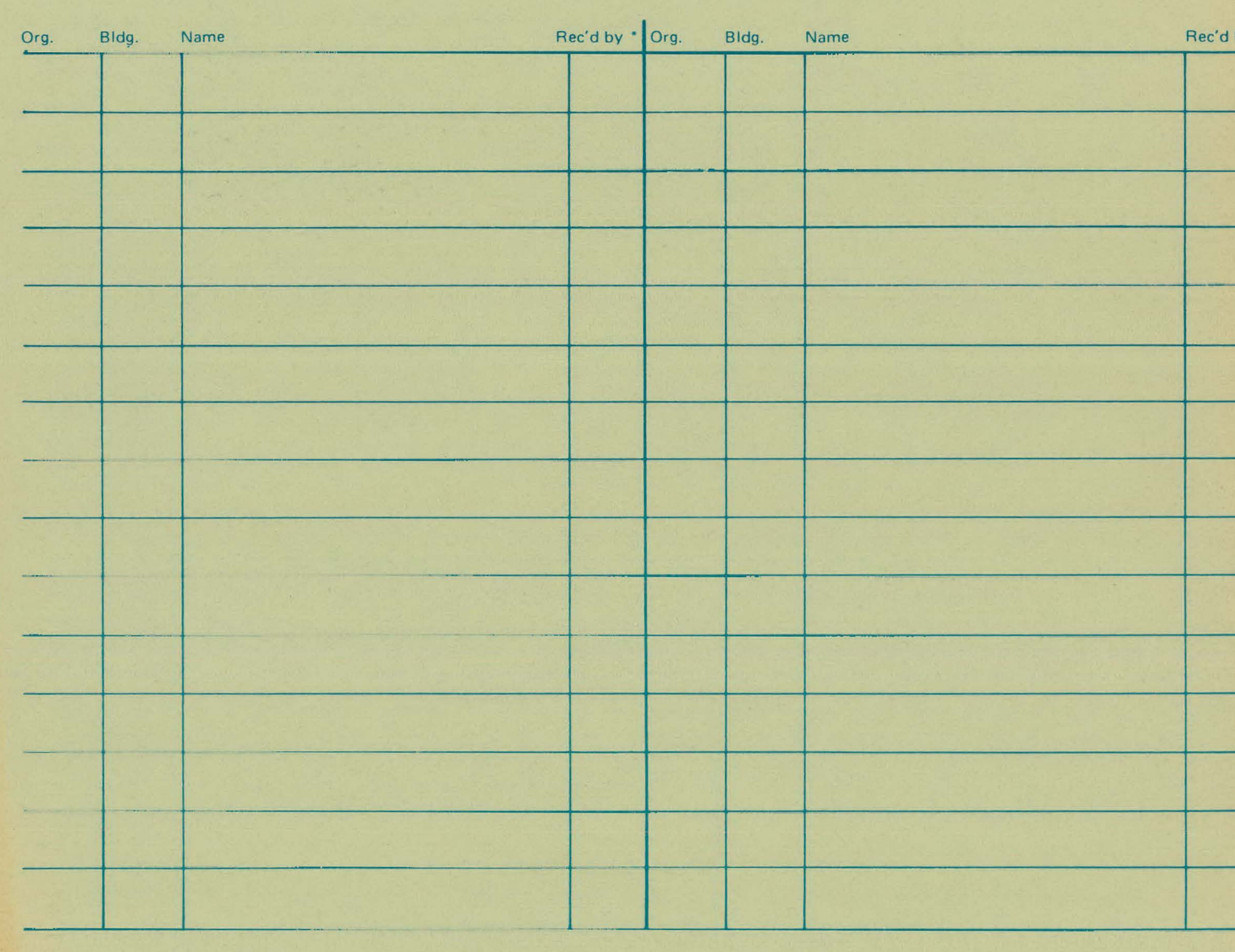

